\title{
Les pagnes des circoncis
}

Séparation et émotions dans les rites d'initiation seereer (Hireena, Sénégal)

Loincloths of the circumcised: separation and emotions in Seereer initiation

(Hireena, Senegal)

\section{Aurélie Troy}

\section{OpenEdition}

\section{Journals}

Édition électronique

URL : http://journals.openedition.org/span/746

DOI : $10.4000 /$ span.746

ISSN : 2268-1558

Éditeur

École pratique des hautes études. Sciences humaines

Édition imprimée

Date de publication : 1 décembre 2008

Pagination : 41-104

ISSN : 0294-7080

Référence électronique

Aurélie Troy, "Les pagnes des circoncis », Systèmes de pensée en Afrique noire [En ligne], 18 | 2008, mis en ligne le 05 juin 2013, consulté le 30 avril 2019. URL : http://journals.openedition.org/span/746

DOI : 10.4000/span.746

(c) École pratique des hautes études 


\section{Les pagnes des circoncis}

Séparation et émotions dans les rites d'initiation seereer (Hireena, Sénégal)

\section{Aurélie Troy*}

Doctorante à l'École Pratique des Hautes Études Centre d'études des mondes africains (CEMAf) CNRS/Université Paris 1/EPHE/ Université de Provence

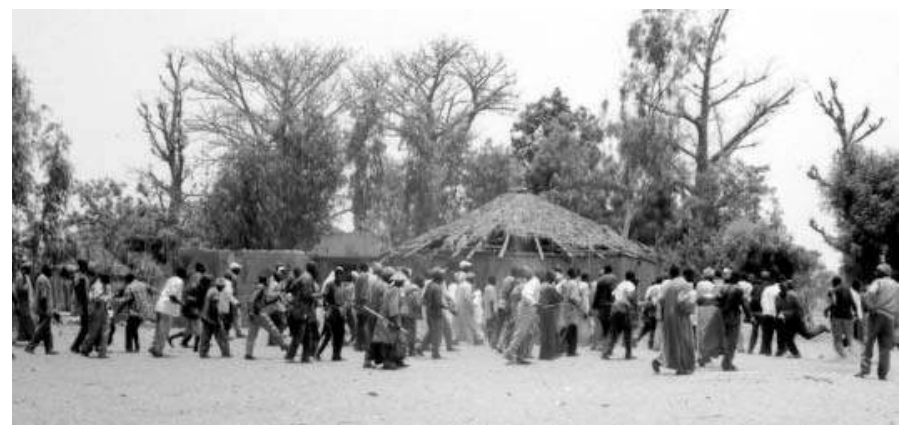

Or nous avons marché tels de blancs initiés.

Pour toute nourriture le lait clair, et pour toute parole, la rumination du mot essentiel ${ }^{1}$

Ce pagne n'est pas un pagne de bambin, c'est un pagne de grand Pourtant la perdrix peut régner, grâce à sa lente démarche

En pays seereer comme dans d'autres régions du Sénégal et de l'Afrique, des tissus que l'on nomme communément pagnes depuis la deuxième moitié du XVII siècle sont employés à des fins rituelles et marquent les étapes décisives de la vie des individus : naissance, initiation, mariage, mort ${ }^{3}$. Pièces de coton rectangulaires composées d'un ensemble de bandes cousues entre elles, dont l'usage vestimentaire est aujourd'hui restreint, ces étoffes composent ou complètent le costume, agrémentent une belle tenue en des circonstances festives. Les pagnes seereer se font aussi langes, voiles ou linceuls. Pagnes blancs (pay njoor

\author{
* Je dédie ce texte à \\ la mémoire de Boucar \\ Basse, chef du village \\ de Yayème (photo ci- \\ contre), qui s'est éteint \\ en juin 2005. \\ 1 Senghor, 1990 : 108. \\ 109. \\ ${ }^{2}$ Chant d'hommes (a \\ kim ndaam), interprété \\ par des initiés de Mar \\ Fafako, recueilli à \\ Yayème, la nuit de sortie \\ du ndut, le 07/04/01. \\ Transcrit par Karamo \\ Diame. Ce chant signifie \\ en substance que le \\ circoncis est considéré \\ comme un homme dès \\ lors qu'il a été voilé du \\ pagne de l'initiation. \\ ${ }^{3}$ Mes remerciements \\ vont aux habitants \\ de Yayème, à Birama \\ Siga Faye, Yacine \\ Diop, Moodi Saabu \\ Dioumasse, Kambul \\ Basse, Sagar Mara \\ Diouf, Xeram Basse, \\ Mama Téning Diom, \\ Ndoffène Fata Diouf, \\ Yandé Douf, Baama \\ Sagar et Faamara \\ Basse, au regretté \\ Bamba Diouf de Djilor \\ Dijak, à Jean-Marie Faye \\ de Ndiongolor ainsi \\ que Karamo Diame \\ pour sa précieuse \\ collaboration dans ces
}


affaires masculines et pour l'aide qu'il m'a apportée lors de la transcription en seereer de plusieurs entretiens relatifs à l'initiation.

\footnotetext{
${ }^{4}$ Afin de faciliter la lecture de ce texte, j'utiliserai par conven. tion le singulier pour l'ensemble des termes vernaculaires employés ici. J'omettrai de la même manière de préciser les affixes de classes des noms employés.

${ }^{5}$ Weiner, 1989 ; Schneider, 1987.

${ }^{6}$ Appadurai, 1986 et Bonnot, 2002.

${ }^{7}$ Coquet, 2001 : 28.
}

ou pay ndan ${ }^{4}$ ) ou pagnes noirs teints à l'indigo (pay 6aal) sont les objets incontournables des rites de passages. Entre ces deux pôles chromatiques varie toute une gamme de pagnes à rayures. On distingue ces pagnes "autochtones " des tissus industriels qui les ont remplacés aujourd'hui comme vêtements (leegos, waaxi, bazin, etc.) en les qualifiant de "pagnes tissés" (pay tiwand). Bien qu'ils soient confectionnés de façon artisanale par des hommes (tisserands tukulëër ou griots seereer), ce sont des objets féminins par excellence : seules des femmes mariées et initiées peuvent en gérer les sorties sur la scène rituelle et cérémonielle. Ce sont elles qui se les procurent et les thésaurisent. Et c'est encore par les femmes, en ligne maternelle, que ces pagnes se transmettent.

L'étude anthropologique des textiles couvre une formidable diversité de registres, comme l'ont montré Weiner et Schneider ${ }^{5}$. L'ethnologue qui s'intéresse à la manière dont les tissus s'acquièrent, se gardent, se manipulent, s'échangent ou se portent, aborde la société qu'il étudie sous un angle qui peut se montrer très fécond. Les tissus ont en somme, en tant qu'objets, une "vie sociale ${ }^{6}$ ". Matière dont les corps s'enveloppent, ils cachent ou révèlent; aisément transportables, ils passent d'une personne à une autre, et parcourent ainsi des itinéraires qui tissent alors de véritables « liens textiles ${ }^{7}$.

C'est l'analyse de liens textiles identifiables dans un contexte particulier - les rites de séparation de l'initiation masculine - qui retiendra mon attention ici. Les pagnes blancs ensevelissent les circoncis seereer, les cachent au regard, le temps des festivités qui inaugurent puis clôturent les rites. Que nous disent ces objets des relations entre les participants dans ce contexte hautement dramatisé qu'est le départ d'un novice à l'initiation? Quelle lecture de ces événements qui suscitent des émotions considérables nous permettent-ils? Que nous révèlent, enfin, ces objets féminins du processus initiatique masculin?

Après une brève présentation de l'institution initiatique seereer, cet article débutera par l'examen de ce que 
Van Gennep appelle "rites préliminaires ${ }^{8}$ " et qui visent à séparer les futurs initiés en passe de devenir des hommes du monde antérieur et familier auquel ils étaient liés, en particulier celui des femmes et des enfants. Je décrirai la façon dont les novices sont arrachés à leurs maisons puis emmenés vers la brousse, en prêtant une attention particulière aux différentes modalités d'interaction entre les différents participants à ces rites publics. Ayant ainsi attiré l'attention sur les pagnes qui voilent entièrement le corps des futurs initiés, je m'interrogerai ensuite sur leur provenance et retracerai les itinéraires qu'ils empruntent du début à la fin de l'initiation. Le cheminement des textiles ainsi mis en lumière fera apparaître comme déterminant le travail rituel des mères des novices. Les actes qu'elles doivent accomplir renforcent et malmènent à la fois la relation qui les unit à leur fils. Se prolongeant sur toute la durée de l'initiation, des séquences de séparation successives brisent et refondent le lien maternel en faisant appel au passé. Jeux sur la re-connaissance, échos lointains de la petite enfance, mise à l'épreuve de la fibre maternelle, permettent que s'instaurent progressivement de "nouvelles formes d'êtreavec l'autre ${ }^{9}$ " entre ces deux acteurs principaux.

\section{Le ndut : I'initiation masculine seereer}

Les rites d'initiation masculine des Seereer du Siin, du Hireena et de la Petite-Côte au Sénégal ${ }^{10}$ ne se distinguent guère, dans leur schéma d'ensemble, d'autres institutions initiatiques africaines, entendues dans le sens de « rites de maturité $^{11}$ " - il ne s'agit pas ici d'initiation à des sociétés secrètes ou religieuses. Le vocable ndut, qui signifie " nid ", désigne à la fois l'enclos initiatique en brousse (le lieu où se déroule la période de réclusion des novices) et l'ensemble des rites. Dans le Hireena et sur la Petite-Côte, ce mot est également employé pour désigner l'initiation féminine dont les rites s'enchâssent dans ceux du mariage, lorsque la nouvelle mariée a rejoint le domicile conjugal (on peut préciser alors ndut rew we : ndut des femmes). Au Sénégal, l'initiation masculine des Wolof, quand elle se pratique encore, semble

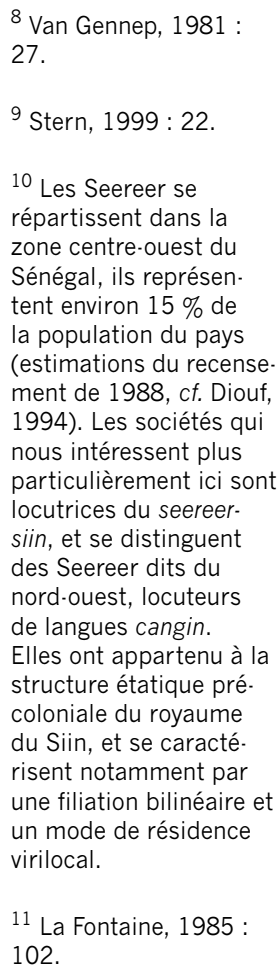


${ }^{12}$ Dione, 1983.

${ }^{13}$ Gravrand, $1990: 40$.

${ }^{14}$ Dupire, 1978; Faye, 1997. assez proche de celle des Seereer : les deux institutions partagent en outre un vocabulaire commun relatif aux différents participants. Pendant le ndut seereer, parmi les chants qui sont produits, nombreux le sont en wolof, ainsi que certaines récitations rituelles. Mes interlocuteurs masculins insistent sur la possibilité de faire rentrer des "étrangers " au ndut : Tukulëër, Wolof, Jóola. Inversement, ils évoquent l'éventualité d'être eux-mêmes, en tant que Seereer, initiés dans les camps de brousse des voisins.

Le ndut est parfois pensé dans la littérature comme le lieu qui dispense une forme d' " éducation traditionnelle ${ }^{12}$ ". Il y est réduit à une «école de formation, pour éduquer l'homme idéal ${ }^{13}$ ». Les auteurs qui l'envisagent sous cet angle se sont surtout attachés à décrypter le symbolisme des chants (vus comme support principal de l'enseignement délivré aux jeunes gens). Les travaux de Marguerite Dupire ou ceux d'Amade Faye ${ }^{14}$ envisagent pour leur part d'autres dimensions $\mathrm{du} n d u t$, et analysent par exemple certaines fonctions rituelles de l'institution. La synthèse qui suit prend certes ces travaux en compte, mais la majeure partie des matériaux qui nourrissent ce texte sont tirés d'enquêtes personnelles, menées dans le Hireena depuis 1996 (voir cidessous cartes 1 et 2).

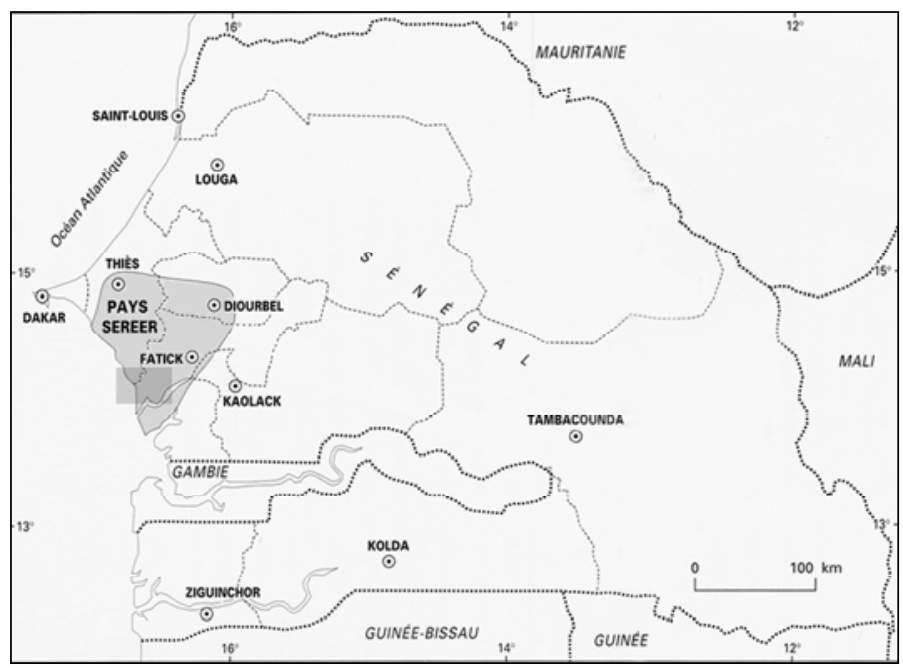

Carte 1- Situation du pays seereer (d'après Léricollais, 1999). 


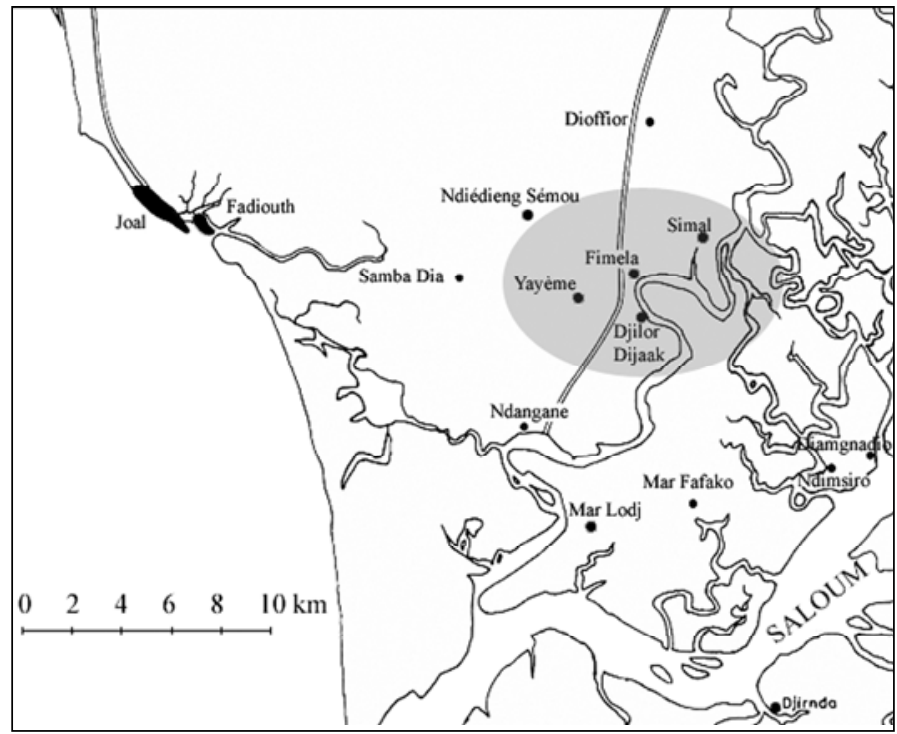

Carte 2 : Situation du Yayème et de la zone d'enquête sur le ndut (d'après Pélissier, 1966).

Autrefois indissociables de la circoncision, ces initiations semblaient se dérouler à intervalles réguliers et réunissaient l'ensemble des jeunes gens d'une à deux classes d'âge. Dans les récits qui m’ont été faits de ces événements, ce sont les incirconcis (xaat) qui estimaient que l'heure était venue pour eux de se faire circoncire. L'organisation d'une session d'initiation n'allait pas sans une suite complexe de visites et de consultations entre parents maternels et paternels des postulants, après lesquelles le roi du Siin - ou le chef d'initiation local (kumax) dans le Hireena - tranchait. Lorsque date était prise, les garçons faisaient connaître leur intention en changeant d'aspect et de costume afin d'informer leur parentèle de cette décision. L'ouverture des rites était alors marquée par des danses qui précédaient immédiatement la circoncision. Délaissées dans le Hireena et sur la Petite-Côte, elles se pratiquent toujours dans certaines zones du Siin. La circoncision avait lieu au village, plus ou moins publiquement. On regroupait par familles paternelles un ensemble de jeunes garçons, et l'opération était effectuée par le circonciseur (yaamaan). Les novices, qui étaient censés prouver leur courage en ne cillant point sous le couteau du vieillard, étaient emmenés en brousse et 
${ }^{15}$ Houseman, 1986a et 1999.

${ }^{16}$ Houseman, 1986b : 38. installés dans le camp où ils demeuraient pendant plusieurs mois. Ils y subissaient les épreuves physiques et les brimades absurdes ${ }^{15}$ bien connues de cette phase liminaire, et on leur soumettait diverses énigmes posées sous forme de chants ou de signes. Une nuit effrayante mettait fin à leur réclusion. L'Ancêtre (Maam), dont la présence se manifestait par le vrombissement des rhombes dans l'obscurité, venait les dévorer. De retour chez eux, ils étaient fêtés et accueillis comme des hommes (goor) qui allaient désormais pouvoir prétendre au mariage.

Aujourd'hui, si le schéma général du déroulement de l'initiation reste à peu près le même, sa durée en revanche s'est considérablement réduite. Bien que le ndut s'étende encore sur toute une semaine dans des villages du Siin, la tendance ailleurs est à la concentration sur trois jours de l'ensemble des rites (départ, réclusion et retour au village). Les hommes évoquent à cela plusieurs causes - colonisation, scolarisation, exode rural, islamisation ou christianisation et déplorent le fait que cela entraine des conséquences fâcheuses pour les nouvelles générations. Regardant en effet le ndut comme lieu de transmission d'un code de conduite moral associé à un corpus de chants, d'énigmes et de danses, ils estiment qu'une quarantaine d'heures passées en brousse ne permettent pas aux néophytes d'acquérir suffisamment de "savoir" (xam-xam). Ces considérations ne leur font toutefois pas remettre en cause l'efficacité ou la force du rituel : l'essentiel reste d'y avoir participé. Être enfermé une première fois et quelques heures au ndut suffit à faire du novice un initié et du garçon un homme. C'est en participant régulièrement aux initiations ultérieures que celui qui le désire pourra augmenter ses connaissances et gravir les échelons qui seront susceptibles de faire de lui un initiateur. Ainsi, un trait remarquable de ce type d'institu-

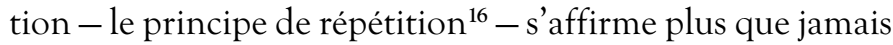
comme aspect essentiel et constitutif du ndut seereer.

De la même façon, les cycles initiatiques auxquels se réfèrent les anciens sont perturbés. De petites initiations demandées par des pères de famille isolés peuvent côtoyer, devancer ou suivre de près de grands ndut. D'un endroit à l'autre du pays seereer, les intervalles entre deux sessions 
d'initiation varient fortement. Certains villages, même, n'en ont plus organisé depuis vingt ans. Lorsque les ndut se font rares, et qu'une session est annoncée quelque part, affluent alors de tout le Hireena de jeunes novices " étrangers ». On dit d'eux qu'ils ont " fui » leur village. Ces "fugueurs " ont bravé les avis parentaux et mis toute leur parentèle devant le fait accompli. Imposant leur présence aux initiés, ils courent le risque, lorsqu'ils arrivent tardivement à l'enclos, de s'en voir refuser l'entrée pour toujours et de tâter sérieusement de leurs bâtons.

Notons enfin qu'à l'heure actuelle, circoncision et initiation ne vont plus toujours de pair. Les garçons, opérés de plus en plus précocement, ont dans la plupart des cas été confiés aux infirmiers des dispensaires, à la discrétion des parents : on circoncit ainsi des bébés comme des garçonnets de trois à dix ans. Rares sont maintenant les puurur (circoncis mais non initiés) qui peuvent se vanter d'avoir affronté le couteau du yaamaan. Ces transformations récentes créent une hétérogénéité certaine au sein du groupe des novices puisque de frêles garçons peuvent côtoyer au ndut de robustes adolescents. Avec de tels écarts d'âge, les novices (njuli) ne sont donc égaux ni physiquement ni intellectuellement devant les épreuves qui les attendent. Il est en outre fréquent de voir "partir en brousse " les membres d'une même fratrie (frères de même père ou de même mère), ce qui était plutôt rare il y a cinquante ans.

\section{Distribution des rôles au ndut}

On peut distinguer plusieurs grandes catégories d'acteurs selon leur degré d'implication dans les rites masculins en prenant garde toutefois de ne pas en faire un modèle figé. Les données qui sont synthétisées ci-dessous sont propres à une localité donnée, Yayème, et sont datées : elles résultent d'observations remontant aux mois d'avril 2000 et 2001.

\section{Les initiés et les initiateurs}

Ce que j'appelle "collège initiatique" se compose donc du kumax du village, d'un kalma et du yaay ni juul. Le kumax est le chef de l'initiation, celui sur qui repose en 
dernier recours la décision de tenir ou non une session. Ce personnage accède à sa charge en démontrant ses capacités à lutter contre la sorcellerie, singulièrement lorsqu'il en vient à terrasser "mystiquement" son prédécesseur. Les prétendants à la charge de kumax s'affrontent en effet lors de joutes nocturnes et oniriques sans merci. Le vainqueur de ce combat, celui qui aura su démontrer qu'à sa force occulte personne ne résiste, est alors jugé compétent par les villageois pour devenir à son tour chef de l'initiation masculine. À la fois sage et clairvoyant, il doit protéger le camp d'initiation de toute intrusion sorcière. La survie des njuli dépend de lui. Il ne passe que peu de temps en brousse et " travaille " la nuit, de chez lui. Comme d'autres maîtres de la tête (yaal xoox) en pays seereer, il est de ceux qui détiennent la connaissance de la nuit ( $o$ and $o$ yeng). C'est en rêve qu'il se rend au ndut, démasque les sorciers qui le menacent, leur livre bataille, et veille à ce qu'il n'y ait aucune victime parmi les novices.

Le kalma, qui, lui, accède à sa charge de façon héréditaire (en ligne utérine), seconde le kumax dans cette tâche. On les voit ensemble parcourir le village en tous sens, l'air absorbé, tandis que l'on se saisit des novices le matin du premier jour. Le kalma est aussi un spécialiste des poisons et se charge de la cueillette de végétaux utiles lors des rites de séparation. Il détient en outre de petits bâtons réputés neutraliser immédiatement les substances nocives que des êtres malveillants auraient pu mêler à la boisson ou aux aliments des njuli.

C'est à celui que l'on appelle yaay ni juul, littéralement, mère des circoncis, que l'on confie les précieux bâtons-antidotes. Apparenté au kalma en ligne utérine par son père, il les maniera pendant toute la durée du ndut. Mais son rôle ne s'arrête pas là. Comme l'indique son titre, en l'absence de femmes dans l'enceinte du ndut, il doit se substituer aux mères de chacun des novices en les protégeant personnellement. Il intervient dans l'enclos lorsqu'il considère qu'un initiateur abuse de son pouvoir. Il est secondé dans ses tâches par deux ou trois petits frères classificatoires, membres du même segment de lignage maternel et dotés des mêmes pouvoirs. 
Se mêlent ensuite à ces grands initiateurs locaux d'autres dignitaires occupant des charges similaires dans leurs propres villages. Ainsi, se rendent à Yayème les kumax, kalma, yaay ni juul de Fimela, Djilor, Simal, Dioffior ou Mar parfois. Ces personnages se consultent plusieurs jours avant le début de l'initiation, et participent activement à ses rites préliminaires. Notons enfin la présence des yaamaan, ces circonciseurs traditionnels qui, bien qu'ils aient perdu l'essentiel de leurs prérogatives passées, gardent, nous le verrons, un rôle primordial lors de la phase de séparation des novices d'avec leur milieu familial. Outre ces responsables du bon déroulement de l'initiation, l'organisation d'un ndut donne l'occasion à des spécialistes en tout genre de se retrouver, de se défier, d'étaler en public leur science. Aussi n'est-il pas rare de voir défiler, dans les processions qui entraînent les novices vers la lisière du village, des hommes brandissant des lances (salma) et des ceintures magiques (tafu). Ils arborent des chemises bardées d'amulettes, aspergent le sable foulé par le cortège de puissantes décoctions, et profèrent à la ronde leurs prédictions. Ils sont devins (saltigi), voyants (madag), guérisseurs (pan) ou responsables de culte (yaal pangool).

Tous les hommes initiés du village ou apparentés aux njuli peuvent participer au ndut. Ils ne sont pas forcément actifs en tant qu'initiateurs, mais vont et viennent entre brousse et village, tantôt sérieux, tantôt hilares, sanglés de ceintures de coton tissé (seejor), véritables boucliers contre les périls qui les guettent tous pendant ces jours de grande agitation. Viennent ensuite ceux que nous pourrions appeler les "surveillants " ou " moniteurs " des novices. Ce sont les selbe. Initiés depuis plus ou moins longtemps - on désigne les selbe "confirmés " par l'expression selbe mbax -, ils encadrent de petits groupes de novices et sont les véritables initiateurs. Ce sont eux qui mènent la vie dure aux candidats, les menaçant sans cesse de leurs longues badines aussi terribles que des fouets. Ils peuvent souiller leur nourriture et leur infliger toutes les vexations et brimades imaginables dans ce contexte. Ils leur soumettent des énigmes sous forme de chants et les interrogent ensuite sur leur signi- 
fication. Les selbe affluent de toute la région lorsqu'une initiation est annoncée : ils jouissent d'une grande mobilité et s'octroient le droit de taquiner, voire de terroriser les noninitiés - en particulier les jeunes femmes - qui croisent leur chemin. Lorsque s'élèvent leurs badines, mieux vaut s'agenouiller et implorer leur clémence d'un «woor! » (" pitié! ») si l'on veut éviter les coups.

Parmi ces moniteurs, certains cumulent également la charge d'" esclaves rituels " (fad goor we). Un esclave est attaché à un novice particulier dont il est le cousin croisé patrilatéral. Bien qu'étant son supérieur hiérarchique du strict point de vue de l'initiation, puisqu'il est selbe et initié lui-même, il est entièrement dévoué à son jeune " maître " (yaal). Il veille sur lui, l'assiste et le sert. De nombreux fad' font ainsi la navette plusieurs fois par jour entre le village et l'enclos de brousse pour apporter les plats élaborés par leurs homologues féminins : les esclaves rituelles ( $\mathrm{fad}$ rew we). Quant aux garçons à peine initiés, si l'on peut dire, et qui participent pour la deuxième ou troisième fois de leur vie à une initiation, ils ne deviennent pas automatiquement selbe mbax. Durant une ou deux sessions, ils passeront par l'échelon intermédiaire de selbe "poussin " (selbe cuuc). Au cours de cette période, ils ne seront pas actifs en tant qu'initiateurs, mais seront, en définitive, les véritables destinataires des " enseignements " du ndut. C'est en effet à eux que les aînés plus expérimentés s'adressent réellement lorsqu'ils lancent des chants-énigmes. Les selbe cuuc sont donc en phase d'apprentissage actif. Dans le même temps, ils sont encore l'objet d'attentions de la part d'initiateurs plus âgés qui les guident et les conseillent. Initiés inachevés, ils doivent en quelque sorte être encore couvés ${ }^{17}$.

\section{Les non-initiés}

Les non-initiés - ces victimes plus ou moins complices des manœuvres de dissimulation des hommes initiés ${ }^{18}-$ et

17 La Fontaine, 1985 : 125.126.

18 Houseman, 1993. qui prennent part aux rites publics de l'initiation, se répartissent en plusieurs ensembles qu'il est important de distinguer. On trouve d'abord, tout en bas de l'échelle, les 
petits enfants des deux sexes. Les petits garçons sont des spectateurs particulièrement concernés par ces cérémonies de départ et de retour du ndut: ils n'ignorent pas qu'ils connaîtront le sort des njuli un jour. Si, parmi eux, certains sont déjà circoncis, d'autres ne le sont pas encore.

Du côté des femmes, il convient de distinguer les femmes initiées de celles qui ne se sont pas encore mariées suivant le rite seereer (ngulook) et qui n'ont par conséquent pas encore subi l'initiation féminine (ndut rew we) : jeunes filles - futures épouses possibles des njuli - ou femmes mariées de tous âges mais n'ayant pas rejoint la maison de leur mari. Le fait qu'elles soient ou non initiées au ndut des femmes place d'emblée ces personnes dans des positions structurelles différentes par rapport à l'ensemble des phénomènes de dissimulation qui se mettent en place au cours du rituel. Parmi les femmes initiées, il existe en outre une hiérarchie discrète qui fait écho à celle qui prévaut pour le ndut masculin. D'apparence moins structurée, l'institution féminine est néanmoins pareillement dotée d'une élite d'initiatrices. La reine des femmes (maad ni rew we), comparable au kumax, est élue à sa charge, tandis que ses suivantes (jaraaf) héritent en ligne utérine de leurs fonctions.

Les novices, enfin, sont des candidats plus ou moins volontaires, souvent résignés, dont les parents cautionnent (et financent) la participation à cette session. Tous égaux devant le $n d u t$, ils seront cependant distingués par leurs initiateurs. En effet, ils se verront attribuer des surnoms traduisant ou induisant des rôles ou qualités. L'un d'entre eux sera désigné « chef des njuli » par les anciens, c'est le dok. On lui reconnaît des qualités exceptionnelles, de celles qui font les grands initiateurs futurs. Les autres postulants que sont ces novices «fugueurs " dont je parlais plus haut sont rarement visibles lors des rites de séparation puisqu'ils se rendent directement au camp de brousse. Objets du rituel destiné à faire d'eux des adultes, les novices quels qu'ils soient n'en sont pour ainsi dire jamais sujets. Comparés aux selbe cuuc, les njuli semblent tout à fait passifs. Ils subissent leur initiation plus qu'ils n'y prennent part en tant qu'agents. 


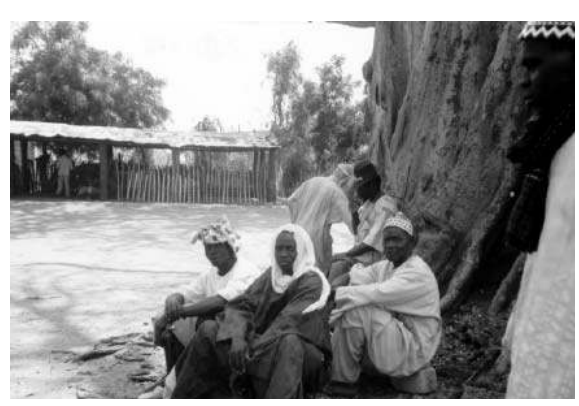

1. Membres du collège initiatique (Yayème, 2001)

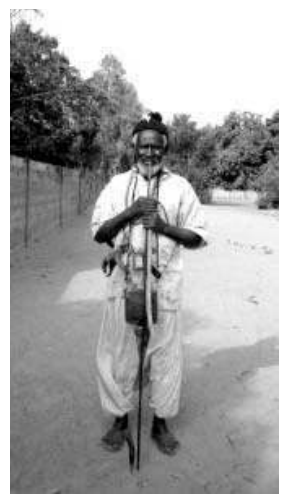

2. Le kumax de Yayème (Yayème, 2000)

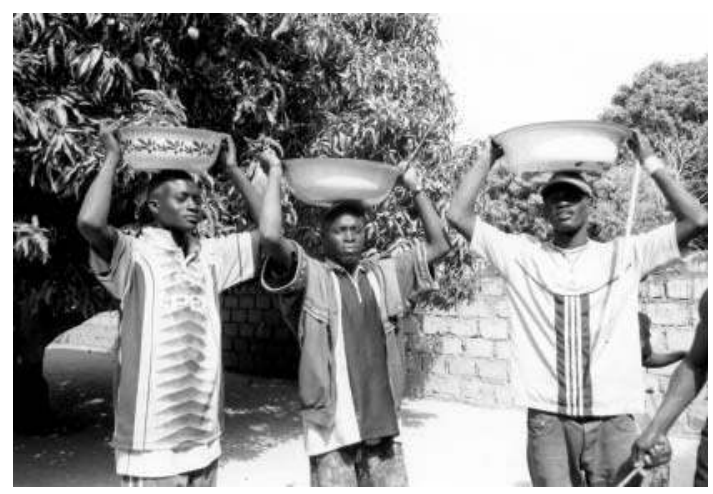

3. Des esclaves rituels prêts à emporter des plats au ndut (Yayème, 2000)

\section{Le départ des novices}

Les rites préliminaires du ndut sont en partie publics et se déroulent le matin du premier jour. Plusieurs événements marquent cette phase qui verra les novices rejoindre le camp de brousse. À l'aube, chaque garçon est lavé par son père, puis isolé dans une pièce de la maison dans laquelle il recevra un dernier repas de sa mère. Cette dernière, ou une tante paternelle, l'aspergera d'un mélange de poudre de feuilles de baobab (laalo), de sel, de farine de mil avant qu'il 
ne soit voilé du pagne blanc de l'initiation. Ainsi dissimulés, guidés par leurs initiateurs, les njuli débuteront leur marche vers la place publique dont ils effectueront des tours en file indienne et sous la bonne garde des selbe armés de bâtons, avant d'être finalement éloignés des habitations et conduits dans la forêt.

Au petit matin, des visiteurs commencent à affluer des villages alentour. Une agitation peu ordinaire monte dans les allées. À l'intérieur des maisons concernées directement par l'organisation du ndut, celles où l'on doit prendre des novices, l'affairement est d'une autre nature. Les activités habituelles sont perturbées. Les enfants n'ont pas leurs jeux coutumiers et vont par petits groupes silencieux et discrets; des jeunes femmes finissent de balayer la cour; d'autres plus âgées vont et viennent en silence, cachant parfois des ballots de tissu sous un pan de foulard.

Chez Boucar, on a regroupé huit garçons de la même famille paternelle. Devant la porte principale, les griots venus du village voisin retendent leurs peaux et affûtent leurs baguettes. Pour eux, la matinée s'annonce bien remplie. Ils suivront bientôt des responsables de l'initiation (kalma, yaay ni juul), d'autres initiateurs (selbe), et des initiés qui pénètrent dans l'habitation en un flot discontinu et se dirigent vers le fulang, petite cour située au fond de la concession et où l'on procède d'ordinaire aux ablutions corporelles à l'abri des regards. Certains initiés pénètrent dans cette enceinte rituelle tandis que d'autres se postent à l'entrée et en délimitent physiquement le contour.

Pendant ce temps, les femmes de la maison accueillent dans une grande discrétion leurs amies ou parentes concernées par la cérémonie. Elles s'installent sur de petits bancs qu'elles ont apportés, s'agglutinant les unes aux autres, le long de la maison principale de la concession, faisant face à la cour. Il est frappant de constater qu'elles s'arrangent ici comme si elles devaient assister à quelque divertissement (des danses par exemple), alors que précisément, de la façon dont elles se placent, à l'écart de l'agitation masculine, on comprend qu'elles ne verront rien. 
Je réalise bien vite qu'elles vont être, en somme, les spectatrices impuissantes d'un spectacle auquel elles n'assisteront pas : elles n'ont rien à voir dans ces affaires d'hommes, littéralement, ou plus exactement pas ce que l'on imagine. Entre elles et les novices, les hommes debout, formant des grappes compactes, leur tournent le dos. Assises au ras du sol et loin derrière, elles ne voient que leurs jambes. Anxieuses, silencieuses, les plus âgées d'entre elles sont graves. Alors que certaines, plus jeunes, se permettent d'échanger quelques mots ou même de rire, l'attitude soucieuse des "mères" tranche d'emblée avec celle des « sœurs » ou amies.

Plus le temps passe, plus l'attente se prolonge et plus les visages des femmes se ferment. Certaines ont la tête dans les mains, et y cachent une larme. En face d'elles, de l'autre côté de la cour, de jeunes garçons rasent les murs, se groupant dans l'ombre. Eux d'ordinaire si vivants se tiennent cois. Fascinés, ils fixent la masse des hommes qui barrent maintenant l'accès au fulang, visiblement impressionnés. À distance enfin, quelques fillettes vont deux par deux, entre amies, et observent les garçons. Amusées et intimidées tout à la fois, elles commentent à voix basse les événements. À côté de moi, je saisis un murmure. Une femme soupire : "xaye tee dom, de! " : Ça va être dur aujourd'hui, dé!"

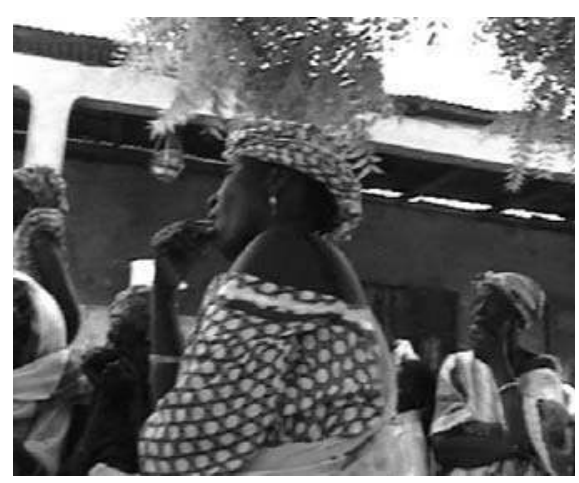

4. Attente des mères (photos extraites d'un film - Yayème, 2001) 


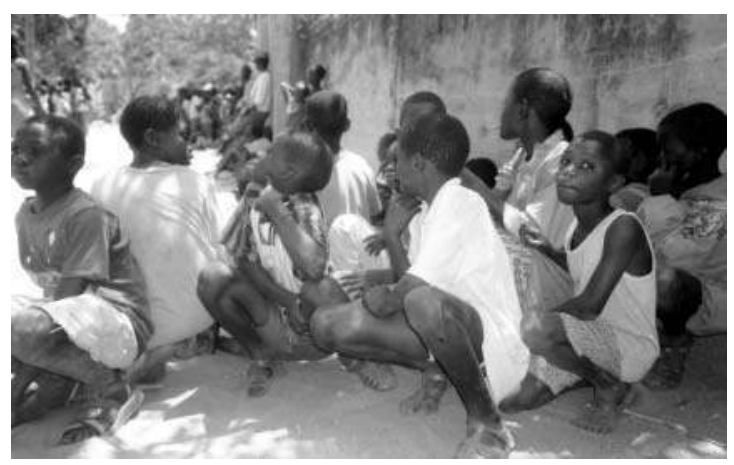

5. Attente des cadets des novices (Yayème, 2001)

Pour l'observatrice que je suis, le contraste entre les attitudes de ces différents participants est frappant : tout se passe comme si les émotions des uns nourrissaient en retour les comportements des autres. Devant un public qui retient son souffle, les hommes initiés vont et viennent à leur guise, arborant fusils et bâtons, parés d'impressionnantes ceintures d'amulettes. La journée leur appartient, ce rituel est le leur. C'est leur rôle que d'impressionner les femmes, et ils n'hésitent pas à en rajouter : «a mbi'a ngoora den " ( ils font leurs trucs masculins »), me dit un ami. Les non-initiés quant à eux, sont aussi silencieux, tendus et statiques que les initiés sont expansifs, bruyants et libres d'aller et venir. Cloués sur leurs bancs ou adossés aux murs, ils s'effacent.

La tension devient maintenant palpable. Une rumeur prend naissance dans le fond de la cour et gagne l'audience qui en est exclue. Un frisson parcourt l'ensemble des participants et le silence se fait. Soudain, du cœur de l'enceinte rituelle, une première devise tambourinée claque. Elle est aussitôt accompagnée d'un coup de feu. Les non-initiés sursautent et se crispent. On a voilé le premier novice! Ces couples sonores explosifs (tambours/fusil) se succèdent autant de fois qu'il y a de candidats au ndut. Mais que se passe-t-il du côté des hommes? Comment se déroule cette étape cruciale de la séparation qu'est le recouvrement rituel des circoncis (muur)? Si la veille du ndut encore je pensais naïvement que je pourrais assister à la "prise de voile » des 
19 Birama Siga Faye, dont le rôle en tant que " grand initiateur » était celui de mère des circoncis (yaay ni juul) à Yayème, le 21/04/01.

20 «xedaan o reffa jaam, gat fa jaam, xedaan ne paa' nee nannaa xoox lee wo rangiit neen, xedaan $u$ mof boo muura o beef $»$.

21 Zempléni, 1991. njuli, une fois assise auprès de leurs mères, j'ai dû renoncer à ce projet. Sur le moment, j'en étais réduite à laisser faire mon imagination (et à être prise personnellement dans le piège émotionnel de cette mise en scène). C'est quelques jours plus tard que je commençai une série d'entretiens sur le $n d u t$ avec l'un des initiateurs ${ }^{19}$ de cette session et rassemblai les éléments qui suivent.

Quels sont les acteurs directement concernés par le muur? Ce sont, en premier lieu, les pères (réels et classificatoires) des novices ainsi que les esclaves rituels qui leur sont attachés ( $f a d$ goor we). Le père réel procède à un bain rituel protecteur à base de décoctions de racines. Il confie ensuite deux pagnes blancs à l'un de ses "frères " qu'il aura choisi dans son segment de matrilignage (den) - trajet 1 dans la figure 1 ci-après.

Le fait de choisir ce petit ou grand frère est une façon de lui signifier qu'il n'y a aucun différend entre eux : c'est une marque de confiance car, m'explique Birama Siga, « il est seulement ce que je suis ". C'est vraiment dans sa famille paternelle qu'on inscrit ici le circoncis, ou plus précisément, dans le lignage maternel de son père. Le père classificatoire ainsi chargé de voiler le novice profère une série de conseils et de vœux (aas) où il est demandé aux novices de mesurer le mal qu'on se donne pour eux, et de prendre conscience des dépenses énormes et des sacrifices qu'occasionne à la famille leur initiation. Entre-temps, l'esclave rituel $(\mathrm{fad})$ s'est vu confier (2) le soin d'attacher un premier pagne à la taille du njuli, qui se déchausse, et s'assied sur une natte (3). Le " père "s'empare alors du deuxième pagne, couvre et découvre alternativement la tête du novice, quatre fois (4) en disant : «Puisses-tu être en paix, revenir avec la paix. Puisse ta tête blanchir un jour comme ce pagne blanc. Puisses-tu vivre assez longtemps pour voiler à ton tour, un jour, ton propre fils d'un pagne ${ }^{20}$." Il souligne ainsi le nécessaire caractère transitif ${ }^{21}$ de l'initiation tout en émettant un vou de longévité. La blancheur du coton dont est tissé le pagne est en effet fréquemment associée dans les rites seereer à la blancheur des cheveux. 


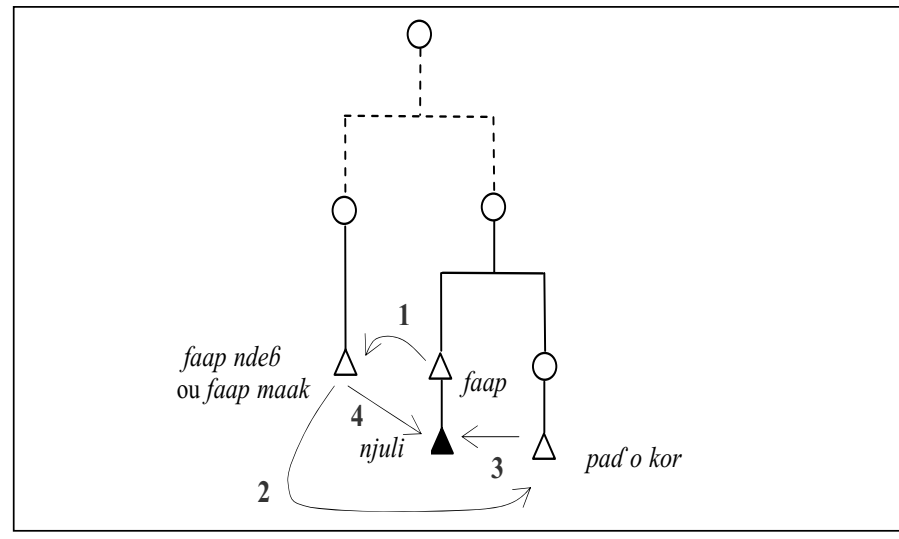

Figure 1 - Personnes impliquées et trajets des pagnes blancs dans

le rite de recouvrement des circoncis.

Une précision importante m'est ensuite parvenue. Avant que l'on ne voile les garçons, le circonciseur traditionnel (yaamaan) intervient. Quand bien même les njuli ont tous été circoncis longtemps avant les rites, au dispensaire (dans la plupart des cas), ce personnage surgit, couteau à la main, et va se livrer à une pantomime évocatrice : il entre, sort de l'enceinte rituelle, recommence. Il brandit ses attributs (couteau, amulettes), trace des lignes au sol, crache, met une pincée de sable sur le front du novice et tranche un prépuce imaginaire. Quand il a fini, le tambourinaire (paar) bat le tambour et l'on voile enfin le novice. On tire un coup de fusil et, me dit-on, "les hommes et les femmes à côté savent ainsi que l'un des njuli est circoncis ». Pour les initiés qui me rapportent ces actions et les critiquent, le yaamaan " ouvre la coutume " (a weeta mbax), tout est "démasqué " (a weeja). Des faits comparables ont été rapportés par Droogers ${ }^{22}$, qui signale un simulacre de circoncision au début de l'initiation pour ceux qui ont été opérés à la clinique. L'action de ce personnage qui semble jugée superflue par certains commentateurs locaux (puisqu'il n'agit plus "pour de vrai ") me paraît au contraire encore tout à fait nécessaire. Circoncision ou pas, on ne peut se passer de son intervention lorsque l'on vient chercher les novices : il reste un agent essentiel de la phase de séparation.

On a donc une première caractéristique de cette étape du rituel : elle consiste en la mise en scène d'une circoncision symbolique. Les novices sont voilés par petits groupes

22 Droogers, 1980 :

125. 


\footnotetext{
${ }^{23}$ Gravrand 1990 : 52; Dupire, $1978: 117$.

24 Petit arbuste de

la famille des

Combrétacées (Crétois, 1972-1977).

${ }^{25}$ Plante herbacée

de la famille des

Asclépiadacées.
}

de la même manière qu'ils étaient circoncis à l'époque ${ }^{23}$. Notons d'ailleurs que le vocabulaire employé pour désigner ce jour de départ des novices reste lié à la circoncision. On dit : « ngoor ke naa nguckeel xaye » : « les garçons se font circoncire aujourd'hui. " Le recouvrement rituel et la circoncision entretiennent ainsi un rapport métaphorique. En outre, la façon dont chacun des non-initiés vit le muur laisse entendre que les choses sont prises très au sérieux. Paroles inaudibles, gestes cachés, njuli inaccessibles aux "spectateurs", sons des tambours et coups de fusil, allées et venues des initiés : autant d'éléments qui alimentent l'intensité dramatique de cet épisode rituel et qui contribuent à ce que l'on tienne cette circoncision - toute symbolique qu'elle soit - pour réelle. On valide enfin une opération passée sous silence, celle du dispensaire, à laquelle des femmes ont d'ailleurs pu prendre part. Pendant le muur, les hommes manipulent les pagnes, les novices et les non-initiés, en s'appropriant des gestes qu'ils réinscrivent dans un contexte masculin et une filiation paternelle.

\section{Du seuil à la lisière : espaces divergents, regards croisés}

Les novices désormais voilés, méconnaissables, sont guidés par les hommes vers la sortie de la concession. La société seereer, comme tant d'autres en ces circonstances, marque tout particulièrement les frontières, les limites. Ainsi, la porte de la maison a été soigneusement préparée pendant qu'au fond de la cour, on procédait au muur. Aux lignes qui s'entrecroisent, tracées dans le sable devant le seuil par le kalma, s'ajoutent de longs fagots de branches. Les essences employées ici se retrouvent à l'occasion du rite de sortie de case d'une jeune accouchée. Il en est ainsi du yaay $^{24}$ et du mbodaafod ${ }^{25}$. Les vertus du yaay sont liées à la reproduction; ses branches assurent une longue descendance. Quant au mbodaafod, le poison que sécrète ses racines a la faculté d'éloigner génies et sorciers. Comme si ce premier ensemble de seuils symboliques ne pouvait suffire à marquer la séparation, les initiateurs renforcent encore ce dispositif : deux tiges souples accolées en leurs extrémités 
forment, lorsqu'on les écarte, un étroit passage à travers lequel les novices, aveuglés sous leurs pagnes, devront se faufiler sans tomber. Dans les descriptions de Dupire et de Gravrand $^{26}$, on retrouve ce cerceau ellipsoïdal, mais c'est à la porte de la case de la mère ou du père qu'il est franchi par le jeune garçon en route pour la circoncision. On le désigne du nom de l'arbuste dont il est issu : ñaalafun ${ }^{27}$. Ce sont les yaay ni juul qui vont le chercher en brousse. L'un d'eux me rapporte : "Lorsque tu passes à travers, au milieu de cette ouverture, rien ne peut plus t'arriver! Aucune "mauvaise personne" (kiin o paxeer) ne pourra s'emparer de toi pour te faire du mal. "

Pour Dupire, ce rite symbolise clairement « la renaissance sociale [que le novice] va subir ${ }^{28}$ ". Pour ma part, j'interpréterais plutôt ce passage comme figurant une naissance inversée, une dé-naissance; un acte rituel propre à dé-faire la naissance nécessairement maternelle et féminine d'un garçon. Lorsque les novices voilés sortent de leur maison, ils enjambent un entrelacs complexe de lignes horizontales. Mais en se faufilant à l'aveugle dans cet orifice qui évoque une vulve à laquelle aurait été attachée une valence masculine, matérialisé dans l'espace par le ñaalafun vertical, ils entrent simultanément dans une nouvelle dimension. Empruntant ainsi un chemin inverse à la naissance, ils s'en retournent vers une matrice masculine cette fois (rappelons que ndut veut aussi dire "nid" en seereer), d'où ils ne sortiront qu'à l'issue d'une gestation symbolique plus ou moins longue. La re-naissance sociale a lieu plus tard, au moment du yengdari, la nuit de l'ancêtre, où ils seront avalés et régurgités par le monstre puis lorsqu'ils paraitront dévoilés aux non-initiés le lendemain de leur retour au village. Fait récurrent des initiations masculines, où les hommes initiés s'efforcent de se "[réapproprier] symboliquement la reproduction de leurs semblables " pour palier le fait incontournable qu'ils « ne peuvent accoucher ni de leurs opposés ni de leurs semblables, [et] doivent toujours passer par un ventre féminin pour naître et pour faire naître ${ }^{29} \%$.

Tombé en désuétude ou méconnu dans cette frange du Hireena, un épisode significatif de la séparation, systé-

\footnotetext{
26 Dupire, $1978: 116$ 117; Gravrand, 1990 : 52.

${ }^{27}$ Arbuste lianescent, épineux de la famille des Combrétacées : Combretum aculeatum Ventenat (id.)

28 Ibid.

${ }^{29}$ Moisseeff, 1987 :

133.
} 
30 II est également bien documenté dans la littérature. Cf. Dupire, 1978 : 117; Gravrand, 1990 : 54-55; Faye, $1997: 231$.

${ }^{31}$ Gravrand, 1990 : 54.55.

32 Dupire, 1978 : 117.

33 Birama Siga Faye, Yayème le 21/04/2001. matiquement évoqué par mes interlocuteurs, mérite d'être mentionné ici ${ }^{30}$. Il s'agit d'une scène qui se joue entre mères et fils avant que le jeune garçon ne franchisse le seuil de sa maison. On parle de "dernière tétée " (a nuum a paktum). Voici comment le Père Gravrand la décrit ${ }^{31}$ :

[...] il reste encore un acte émouvant à remplir. Le circoncis est censé renaître. Aussi pour symboliser sa vie nouvelle, va-t-il boire une nouvelle fois au sein de sa mère, tourné vers l'orient. Celleci, toujours assise, pleure au milieu de ses amies. Elle découvre devant son grand fils sa poitrine flétrie. Et lui, il fait le geste sacré de boire. La minute est vraiment impressionnante. Le nouveau circoncis se met à l'écart et déjà l'intérêt du public se reporte sur le second qui va être circoncis de la même manière. Le troisième suivra, alors que la nuit commence à tomber.

Après avoir bu au sein maternel, le circoncis va être arraché à sa mère. Il se rend auprès de son père qui trace un cercle autour de ses pieds, ainsi que deux lignes croisées. Par trois fois sa mère l'appelle par son nom. À la troisième, il quitte le cercle, monte sur les lignes croisées avec le pied droit, sans se retourner. Sa mère lui dit "Reti fa jam! ", "va en paix! " Il part rejoindre les autres circoncis au sindnorand pour les soins. "Il est devenu un homme». (Notes du 18 janvier 1952)

Ici, comme dans les récits qui m’ont été faits à Njongolor dans le Siin (car je n'y ai encore jamais assisté), cette tétée rituelle est interprétée comme un ultime sevrage qui «implique [une rupture] avec le monde féminin et sécurisant ${ }^{32}$ \%. À Yayème cependant, il semble que la dernière tétée n'avait lieu qu'en des circonstances particulières. En effet, lorsqu'un enfant rendait la tâche ardue au circonciseur "car son corps était chaud, il avait dansé [...] jusqu'à la fièvre ${ }^{33}$ " et que, forte tête, il se permettait de le tromper en lui présentant un sexe de femme, on l'amenait à sa mère, afin qu'il tète pour casser la magie qui lui faisait tenir tête au yaamaan. Il est couramment admis que le lait de la mère, comme son sang menstruel ou parfois même sa simple présence suffisent à rompre la plupart des sorts d'origine masculine ou à anéantir le pouvoir de certaines amulettes. Cette image qu'offre le rite de l'enfant tétant sa mère, tableau hautement évocateur de la force du lien qui unit ces deux êtres, aurait ici une fonction paradoxale : soumettre le garçon indocile. La mère, en se faisant complice des initiateurs, trahit son fils. La proximité réconfortante 
et sécurisante du corps maternel tel qu'il est perçu dans la petite enfance se transmue en distance. Ce rite opère le détachement qui contraint ensuite l'enfant à se plier à la loi des adultes initiés.

Dans le cours du rituel actuel, c'est donc sans qu'un seul regard ne puisse être échangé avec leurs mères - aucun contact visuel direct n'est désormais autorisé - que les premiers candidats franchissent le seuil de l'habitation. Les initiateurs leur font ensuite suivre un itinéraire déterminé à l'avance dans les allées du village avant qu'ils ne gagnent la grand-place, au centre de laquelle s'élève un majestueux baobab lié au fondateur des lieux. Ce cheminement dans les espaces publics sera ponctué de longues pauses à proximité des maisons d'où seront extraits d'autres jeunes gens qui viendront grossir la cohorte des novices. Au cours de ces moments d'attente, alors que l'on procède encore au muиr dans d'autres maisons, les premiers novices restent debout, immobiles sous leurs pagnes immaculés. Les selbe les ont parqués à l'ombre et éloignent à grand fracas les non-initiés qui seraient tentés de les approcher de trop près. Quand ils atteignent enfin la grand-place, leur groupe, qui s'est formé lentement, s'est également accru de plusieurs dizaines de candidats. Une foule grossissante et joyeuse, majoritairement féminine, les a suivis à distance et va former un arc de cercle aux abords de la place.

Là, les hommes entament de lentes circonvolutions autour de l'arbre fondateur. Les griots sont en tête de la procession. Viennent ensuite quelques danseurs intrépides, puis, dignes et graves, les pères des novices et les grands initiateurs. Le kumax y fait une courte et discrète apparition. Viennent alors les selbe sur deux rangs, encadrant la longue file des njuli sous leurs pagnes. On reconnaît près d'eux, aux nattes qu'ils portent pour leurs "maitres ", les esclaves rituels des novices. Un chant lancinant monte de ces processions masculines : "a ndoo', a ndoosa'! " Sa signification demeure inconnue aux non-initiés et il est propre à la séparation.

À la fin du quatrième tour, au signal des tambours, tous les hommes s'arrêtent. On fait s'agenouiller les circon- 
cis, qui se recroquevillent sur le sol, comme ployant sous le poids de leurs pagnes, dans une position presque foetale. Le silence se fait tandis qu'un orateur offre un dernier bouquet de vœux aux novices. Des coups de fusils mettent un terme à ce discours et déclenchent l'une des danses les plus impressionnantes qu'il m'ait été donné de voir en pays seereer : le sox. Les selbe se déchaînent dans des pas de deux spectaculaires : lançant haut leurs jambes et frappant le sol à un rythme effréné (qui évoque un air de carnaval brésilien), maintenant fermement leurs bâtons au-dessus de la tête, ils semblent s'en donner à cœur joie. C'est le moment que choisissent les initiateurs, profitant de la confusion générale, pour faire se relever les njuli et les diriger en hâte vers la lisière du village. C'est ainsi qu'en 2001, quatre à cinq groupes de près de quarante novices ont été ravis de leur maison et menés au ndut.

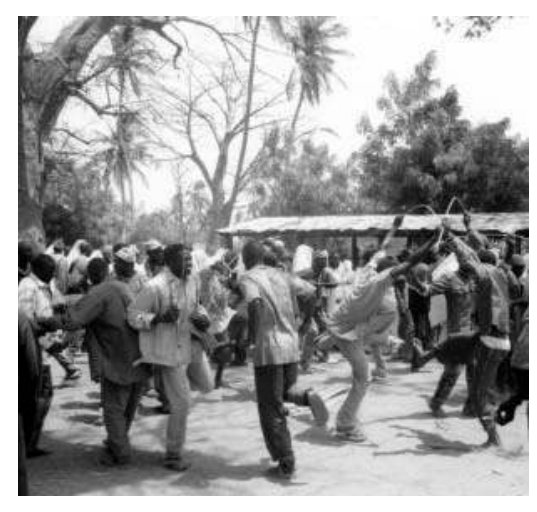

6. Le sox : danse des selbe (Yayème, 2001)

Du lieu du recouvrement rituel des novices à la lisière du village en passant par le seuil de la maison, les participants, selon qu'ils sont initiés ou non, hommes ou femmes, jeunes ou vieux, sont donc plus ou moins tacitement assignés à des espaces distincts. Le rite, en triant les différentes catégories d'acteurs qu'il répartit et ordonne selon un découpage particulier, recompose l'occupation des espaces domestiques et publics du paysage villageois. Des lieux intimes et clos de leur départ (la case du père, le fulang) à la réclusion collective qui les attend au ndut, on fait progresser 
les novices « enfermés " sous leurs pagnes, dans les espaces " ouverts » du village. Sur ce parcours, la répartition des participants évolue et donne lieu à des modalités d'interaction inhabituelles, qui se traduisent par des comportements ou des attitudes plus ou moins stéréotypés. En effet, depuis ces espaces divergents - le fulang, la cour de la maison, son seuil, les allées, les abords d'une place publique, la lisière du village, puis la forêt -, partent et se croisent les regards des uns et des autres sur l'action. En analysant, précisément, la combinaison dynamique de ces différents points de vue ${ }^{34}$, c'est-à-dire en prêtant attention à ce que chacun peut percevoir de l'action et en laisse percevoir aux autres, on entrevoit comment le rituel peut jouer sur les différentes attitudes émotionnelles et rendre alors effective la transformation de certaines relations dont les njuli sont l'objet.

\section{Initiés}

Quels que soient les espaces qu'ils occupent, et on l'a vu pour ce qui concerne le recouvrement des circoncis, les hommes se comportent donc globalement de façon très différente des non-initiés. Mais à l'évidence, leurs attitudes sont loin d'être totalement unifiées et l'on observe des contrastes importants selon les âges ou encore les positions occupées. Les anciens, initiateurs et membres du « collège initiatique ", sont d'un sérieux à toute épreuve. Le kumax, d'un calme qui n'est qu'apparent, s'isole au sein de la foule, le visage impassible, absorbé par ses pensées et son travail. Kalma et yaay ni juul courent d'un seuil à l'autre et se concentrent sur leurs tâches. Quant aux autres initiateurs, de ceux qui, tels les selbe, jouissent de cette liberté de mouvement que nous avons évoquée, ils sont tout entiers absorbés par l'action. D'humeur plutôt joyeuse, ils n'ont aucun comportement agressif ou intimidant à l'égard des femmes ou des non-initiés en général. Lorsqu'ils ne les ignorent pas tout bonnement et discutent entre eux, ils se permettent tout au plus quelques taquineries en arrivant sur la place publique. Dans cette phase préliminaire, ils se posent en responsables des njuli dont ils doivent assurer la survie, et mener à bien le séjour en brousse. Pour ces hommes matu-

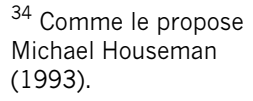


${ }^{35}$ C'est ce mot français « seereerisé » qui est d'ailleurs souvent employé : a mborme'eel, « on les forme ». res, l'enjeu d'un ndut qui démarre est de taille. Les propos qu'ils tiennent à ce moment-là reprennent l'idée qu'il faut à la fois protéger et "former ${ }^{35}$ " les jeunes gens. Aussi, les arracher à leur mère, les tenir sévèrement encadrés par des surveillants prompts à la violence, ou leur faire connaître toutes sortes de privations sont autant de maux nécessaires. L'heure n'est donc pas à la sensiblerie, disent-ils, mais à la rigueur, à l'exaltation des valeurs masculines, à la gravité du devoir qu'ils doivent remplir, tenus qu'ils sont par cette inéluctable triade : le mbax ('obligation rituelle), le kumpa (le secret) et les coosaan (l'histoire, la tradition). Les pères, pour leur part, ne dérogent pas à cette ligne de conduite bien que ces événements aient pour eux une portée particulière. S'ils ne s'impliquent pas tous dans le rituel proprement dit, ils doivent en revanche tous faire face aux lourdes dépenses qu'occasionne l'initiation de leurs fils; c'est une question d'honneur. Au bovin qu'ils devront immoler, s'ajoutent les frais qu'entraîne l'accueil de nombreux visiteurs. Si certains m'ont fait part de la fierté qu'ils avaient à initier leurs fils, d'autres témoignent de l'état de tension dans lequel ils se trouvaient au moment du départ des novices. Car il plane toujours, et pour tous, une légère inquiétude quant au bon déroulement du rituel. S'ils sont émus à la vue de leurs enfants sous les pagnes, ils se gardent bien de le montrer. Seule l'émotion des mères ou de proches parentes est tolérée et attendue. Pères et mères sont ostensiblement séparés pendant cet épisode rituel : leur relation aussi fait l'objet d'une transformation au cours de cette étape. Chacun est nettement dans "son camp "; leur couple est comme suspendu, leur parentalité dissociée.

Autre catégorie d'initiateurs, les selbe sont de ceux que l'on remarque. Grisés par l'ambiance, emportés par l'action, ils agissent de manière parfois très théâtrale. Gestes brusques, conduites effrayantes, irritabilité, arrogance, esprit moqueur; ils ont le goût de la provocation. Leurs bâtons indisciplinés sont là pour impressionner novices, femmes et enfants envers lesquels ils ne montrent aucune empathie. Ils ont un pouvoir visible, et s'en servent, à l'inverse des grands initiateurs dont le pouvoir, invisible, s'exerce à 
d'autres niveaux. À cet égard, leur danse est significative. Le sox est une démonstration de force et de virilité. Mais lorsqu'ils dansent deux à deux et que leurs actes ne sont plus directement destinés aux non-initiés, la sévérité des visages se mue en allégresse. Cette danse collective explose en une dizaine de duels à forces égales. Et malgré les airs de défi qu'affichent les selbe, personne ne terrasse personne; les danseurs affichent ainsi leur égalité devant l'institution initiatique. Leur gestuelle même traduit cette idée (le bâton ne menace pas mais est mis hors d'état de nuire, arc-bouté au-dessus des têtes), ainsi que la composition en miroir du mouvement (voir photo 6). Parmi ces selbe, les esclaves rituels restent relativement discrets, se contentant de guider et de porter les affaires de leurs "maitres". Comme on l'a déjà évoqué, la relation qui les lie aux novices condense deux rapports hiérarchiques contradictoires. Ils leur sont supérieurs en tant qu'initiés; en tant qu'esclaves, ils leur sont assujettis. En outre, en tant que fils de tante paternelle, tout esclave rituel est de même matrilignage que le père du novice. Et, à l'instar de toute personne du matrilignage du père d'un individu, ce cousin croisé patrilatéral est aussi un "père " pour le novice. Le novice est son " enfant ". Cette position ambiguë se lit dans les attitudes que les esclaves rituels prennent ce matin-là. On pourrait parler à leur égard d'une sorte de "neutralité affective " : il leur est difficile de se montrer franchement agressifs envers les njuli - qu'ils servent en tant qu'esclaves et qu'ils protègent en tant que "pères ", comme ils ne peuvent afficher ouvertement de la sympathie à leur égard, surtout aux yeux des non-initiés.

On constate bien que les hommes "fabriquent " ce jour-là de l'entre-soi - quel que soit d'ailleurs le rôle qu'ils jouent dans la phase de séparation - et cela en donnant à voir la supériorité masculine et la cohésion d'un groupe dont les membres partagent une expérience qui exclut de fait une partie de la population. Ils envahissent l'espace domestique jusque dans ses recoins les plus intimes, et s'imposent dans le village. En reléguant les femmes dans des zones autorisées, ils les maitrisent ainsi idéalement et mettent en actes une certaine organisation sexuée de 
l'espace villageois. Partout où ils se postent, leur souci est de se montrer, tout en cachant aux non-initiés les étapes du départ des novices. Ils s'appliquent à étouffer des gestes et des paroles que les non-initiés ne sauraient entrevoir et ne peuvent qu'imaginer. Ils cultivent ainsi le secret dont ils sont eux-mêmes les garants. Les selbe, par exemple, encadrent littéralement les novices, en s'agglutinant au plus près d'eux. Ils cherchent par là à dissimuler ce que les pagnes seuls ne sauraient occulter. En définitive, c'est dès l'épisode du muur et bien avant la retraite en brousse, que les hommes rendent les njuli inaccessibles et les inscrivent dans un ailleurs indéfinissable.

\section{Non-initiés}

Dans cette catégorie, considérons dans un premier temps ces jeunes garçons, cadets immédiats des novices et qui seront un jour emmenés au ndut dans les mêmes conditions. D'abord maintenus à l'écart du recouvrement des circoncis, ils se tiennent silencieux dans la cour, à l'opposé des mères. Cette situation d'exclusion les rend nerveux. Ils en sont affectés comme les femmes qui leur font face, mais d'une toute autre manière. L'inquiétude qui les gagne a une autre portée: il s'agit que cette scène les frappe, marque leur esprit. À partir du seuil, en revanche, on leur accorde de suivre de plus ou moins près les njuli, ce qu'ils font en silence : une certaine tolérance existe donc à leur égard. Pendant ces longues stations debout, on les laisse quelquefois côtoyer le groupe. Ils affichent alors des mines sérieuses et sont aux aguets; ils jettent à la dérobée quelques coups d'œil à leurs aînés voilés, mais se donnent difficilement une contenance. Un certain malaise est perceptible dans leurs gestes. Les postures qu'ils adoptent traduisent leur perplexité : mains sur la tête, sur une hanche, ou encore portée au menton.

S'ils les laissent s'exprimer lorsqu'ils échangent quelques confidences, les selbe savent aussi leur rappeler qu'ils ne sont que tolérés à leurs côtés. Et de fait, il arrive qu'un sifflement de fouet se fasse entendre, provoquant la dispersion des jeunes gens visiblement effrayés. Lorsque le cortège 
atteint la place publique, les cadets sont les seuls non-initiés qui se risquent réellement à proximité de la lisière du village. Ils sont ainsi aux premières loges lorsque débute le sox des selbe et sont témoins du départ des novices en brousse. L'énergie dégagée par cette danse et la musique qui l'entraîne, associée à la vision des njuli voilés que l'on emmène au loin alimentent chez eux toutes sortes d'émotions, de phantasmes et d'interrogations. Certains se voient déjà selbe et expriment clairement leur désir de faire partie du rituel. D'autres sont trop impressionnés pour réagir. D'autres encore estiment qu'on les nargue et se sentent blessés dans leur amour-propre, voire humiliés. Comme je l'ai souligné précédemment, il existe de grandes disparités d'âge au sein du groupe des novices. Cela explique en partie le ressentiment qu'ont certains jeunes gens à l'endroit de leur famille qui refuse de les initier cette année-là, tandis qu'une grande partie de leurs camarades d'âge part au ndut sous leurs yeux. Le xaat ainsi délaissé enrage parfois au point de se fâcher sérieusement avec ses parents. On le remarque au mutisme éloquent dans lequel il a tôt fait de se murer pendant toute la durée des festivités. Cette ambivalence des attitudes et des émotions est spécifique aux xaat et aux puurur, chez qui les initiateurs entretiennent à la fois la frustration et la crainte. Les espaces même qu'ils occupent les mettent dans ce rapport si particulier qu'ils ont avec les évènements. $\mathrm{Ni}$ tout à fait exclus, ni tout à fait pris, ils se sentent ignorés. Et à les écouter, l'appréhension qu'ils ressentent ne concerne pas tant le grand frère que l'on enlève sous leurs yeux, que leur propre personne. En effet, se projetant dans l'avenir, certains anticipent déjà la peur qui ne manquera pas de s'emparer d'eux lorsque leur tour viendra. Mais il leur faut malgré tout rester maîtres de leurs émotions, et ne pas trop laisser croire ni aux camarades dans le même cas, ni aux initiateurs, que l'on est un poltron. Dans ces affaires, le contrôle de soi est de mise car il s'agit aussi de montrer que l'on est fin prêt et que l'on saura être digne de l'épreuve le jour venu.

Intéressons-nous maintenant aux femmes qui suivent le cortège jusqu'à la place publique. Moins directement concernées que les mères par le rituel, elles n'en sont pas 
moins directement visées par les processus de dissimulation et d'intimidation masculines. Elles se maintiennent à distance des groupes de selbe et de njuli mais se permettent de temps à autre quelques encouragements : en chantant, ou frappant dans leurs mains en mesure. Elles se cantonnent aux abords de la place publique et ne la traversent pas. Les visages sont relativement détendus, les discussions vont bon train; l'heure est à la fête. Quelques vieilles provoquent les hommes qui leur passent devant alors qu' ils effectuent leurs tours. Les jeunes femmes semblent relativement plus troublées que leurs aînées par l'atmosphère qui règne ce matinlà. Leurs commentaires sont ponctués ici et là de "Ndey Saan!»: "Mon Dieu!» Elles prennent garde de ne pas empiéter sur la place, qui est réaffirmée de cette manière comme espace masculin. À l'inverse des mères privées du spectacle du muur, les femmes ont dans l'ensemble le sentiment d'assister à un divertissement qui, s'il intimide un peu, a de quoi réjouir. Que perçoivent-elles en réalité du départ en brousse des circoncis? Le cortège s'ébranle lentement autour de la place, bercé par les chants des hommes. Seules les têtes blanches des njuli qui émergent ici et là s'offrent alors aux regards des femmes. Et lorsque les quatre tours s'achèvent enfin, que les njuli s'agenouillent et se prosternent au sol, elles ne peuvent presque plus les voir puisqu'ils sont rassemblés derrière l'arbre tutélaire du village. Débute alors la danse des selbe dont le tumulte et la poussière qu'elle soulève achève de cacher les novices et masque aux spectatrices le fait qu'on les fait se relever en hâte. Le sox détourne ainsi leur attention et permet aux initiés de presser les novices, qui disparaissent en file indienne vers la brousse. L'illusion est totale : les initiateurs escamotent les novices par ce jeu d'écrans.

De telles élaborations rituelles dessinent en creux dans l'esprit des gens une nouvelle construction sociale de la brousse non plus comme lieu familier de culture, d'élevage ou de passage, mais comme lieu interdit et potentiellement dangereux dans lequel disparaissent les njuli. Enfin, lorsque s'achève le sox, les selbe qui dansaient font demi-tour et reviennent en masse vers l'intérieur du village, entraînant 
les femmes et dispersant la foule sur leur chemin. Quand il ne reste plus un seul novice au village, les spectatrices regagnent les maisons où les attendent des réjouissances ainsi que toutes sortes de tâches liées aux préparatifs des repas et au retour des nouveaux initiés.

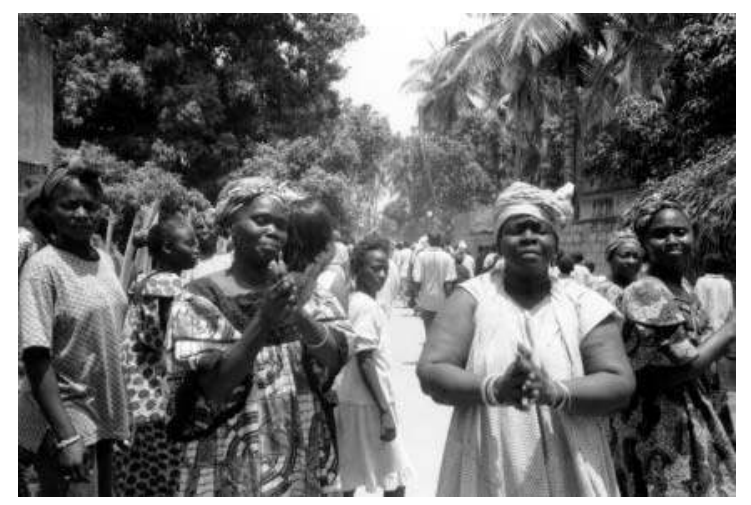

7. Femmes suivant le cortège à distance (Yayème, 2001)

Quant aux mères qui, elles, n'ont pas quitté la maison, on a compris qu'elles n'assistent pas plus au franchissement du seuil de la concession par les novices, qu'elles n'assistaient au muur. En retrait, elles aperçoivent peut-être un court instant les silhouettes de leurs fils qui passent rapidement devant elles, pris en étau dans une cohue d'initiés. Tournant presque le dos à la porte, elles n'auront qu'une perception floue de leur départ. Cette haie d'hommes qui brouille la scène contraint les mères à accepter l'inévitable : la rupture entamée entre elles et leurs fils. Leurs regards sont comme "empêchés "; à l'inquiétude se mêle la résignation. Dès lors que les hommes auront quitté la maison pour la place publique, elles ne garderont avec le rituel qu'un lien auditif qui se fera de plus en plus ténu. Quand la musique et les chants feront place aux rumeurs de la foule revenant de la place publique, elles comprendront que c'est fini. Leurs enfants auront été comme engloutis, absorbés par un monde masculin en effervescence. La séparation physique des mères et des fils aura donc procédé par étapes, comme autant de paliers de décompression. En outre, tout 
${ }^{36}$ Bourdieu, 1982 : 51.

${ }^{37}$ Gravrand, 1973 : 208.

38 " Not-boy-not-man », cf. Turner, $1987: 6$.

39 Ibid. : 7. se passe comme si cette dissimulation progressive des njuli et les émotions qu'elle suscite chez les femmes aiguisaient en retour l'assurance masculine et confortaient les hommes dans leurs opérations de mystification. Les lignes figurées sur le seuil prennent ici tout leur sens. Bourdieu le rappelle : dans ces « rites d'institution ", c'est la ligne et non le passage qui importe. Arbitraire, elle " instaure une division fondamentale de l'ordre social». La séparation ainsi marquée institue " une différence durable entre ceux que ce rite concerne et ceux qu'il ne concerne $\operatorname{pas}^{36} "$.

\section{Les êtres dissimulés}

Dans la phase de séparation, les pagnes dissimulent donc les njuli tout autant qu'ils les désignent. En effet, si l'on ne peut plus voir les novices (leurs corps et leurs visages sont entièrement recouverts), et qu'eux-mêmes ne peuvent plus rien voir (le tissu qui couvre leur visage les aveugle), on ne voit qu'eux: malgré les efforts des selbe, ils attirent le regard et sont finalement le point de mire de tous les participants. Qu'évoquent leurs silhouettes blanches et hésitantes aux spectateurs et, dans le même temps, que perçoivent-ils, eux, de ce moment fatidique où ils sont arrachés à leurs maisons?

L'image des garçons voilés a une forte puissance évocatrice. Pour l'occidentale que je suis, elle évoque des fantômes; pour des Seereer, elle peut évoquer des tig na kob, "choses de la brousse ", ou encore des kuusaasay, des êtres qu'on ne peut pas très bien définir. Notons en passant que d'après Gravrand, la couleur blanche serait chez les Seereer " la couleur des esprits et des puissances spirituelles telles que Djinn, Pangol ${ }^{37}$ ", mais aussi la couleur des morts et des ancêtres. On reconnaît là les liminal personae ou transitionbeings de Turner, qui désigne ainsi les néophytes qui ne sont ni garçons, ni hommes ${ }^{38}$ et dont il dit que «their condition is one of ambiguity and paradox. [...] liminal personae nearly always and everywhere are regarded as polluting to those who have never been, so to speak, "inoculated" against them, through having been themselves initiated into the same state ${ }^{39}$ ". Il semblerait cepen- 
dant que pour les Seereer, avant d'être dangereux pour les autres, les njuli, comme toute personne que l'on recouvre un jour du pagne blanc, deviennent particulièrement vulnérables. Amade Faye rappelle que " parce qu'il peut signifier mort et naissance, [...] le njoor [pagne blanc] a la particularité d'attirer et d'aiguiser l'appétit des puissances nocives; diables, sorciers, serpents, mauvais vents... ${ }^{40}$ ". Les pagnes dont il est dit qu'ils protègent les personnes en passage leur confèrent donc dans le même temps cette fragilité propre aux périodes de transition. Mais indépendamment des dangers relevant de la "surnature " (ou de la nature éventuellement sorcière de leurs propres mères), les novices sont placés dans un état qu'on pourrait qualifier de vulnérabilité sensorielle.

À la simple évocation de l'expérience qu'ils ont eue du muur, de vieux initiés frémissent et serrent les poings en une gestuelle qui évoque celle des lutteurs : émus et heureux, ils se remémorent ces instants qu'ils avouent difficiles avec la nostalgie de ceux qui en sont loin. Lorsqu'ils sont interrogés à ce sujet, les initiés trouvent rarement les mots pour évoquer ce qu'ils ont ressenti sous les pagnes, précisément parce que cette expérience est de l'ordre de l'indicible. À chaque $n d u t$, disent-ils, la vision des garçons sous les njoor leur donne la chair de poule. On dit en seereer littéralement, que ça fait " courir le corps" (cer ke naa yufa).

Après avoir voilé les novices, les initiateurs les dirigent pendant la longue marche qui doit les mener au ndut. Lorsqu'ils franchissent le seuil de leur maison, le passage à travers les branches de ñaalafun implique qu'ils soient guidés et manipulés par les selbe, car aveuglés et empêtrés dans les pagnes, ils ne pourraient le faire seuls. D'ailleurs, quand on ne parle ni du muur, ni de la circoncision pour évoquer toute la phase de séparation de l'initiation, on dit a bood'n ale no juul we, "la reptation des circoncis ". Réduits à l'état de non-voyants qui se suivent les uns les autres en se tenant mutuellement par les pagnes, et guidés par les gestes parfois brutaux des selbe, les novices perdent peu à peu leurs repères. Ce processus de mise en condition qui se met en place aboutit à leur entière soumission. Parfois, les 
41 Birama Siga Faye,

initiateurs s'amusent même à faire tourner plusieurs fois les novices sur eux-mêmes avant qu'ils ne franchissent le seuil de leurs maisons. Cette manière de colin-maillard est reportée à plus grande échelle et renforce le sentiment d'incertitude et d'appréhension quant au sort qui leur sera fait. Les déambulations dans le village et les longues stations debout pendant lesquelles on les fait patienter ainsi que les tours qui sont effectués sur la place publique, les obligent à faire appel à d'autres sens, s'ils ne veulent pas céder à l'étourdissement qui les gagne. Le voile opaque qui s'interpose désormais entre eux et le monde visible provoque donc leur désorientation. Cécité relative, liberté de mouvement réduite et manipulations des selbe, autant de facteurs qui mettent les garçons dans des dispositions émotionnelles particulières. Tout leur échappe, pour ainsi dire, ils n'ont plus prise sur le réel, au point de se sentir perdus dans leur environnement habituel : l'univers le plus familier devient hostile et étrange lorsqu'on vous l'a rendu invisible. Il est bien sûr difficile de dire à leur place ce qu'ils ressentent. Mais dans les témoignages recueillis sur l'expérience du muur, on se rappelle souvent qu'à la fierté ressentie se mêlait un sentiment diffus d'inquiétude. Domine surtout le souvenir d'une grande confusion et d'émotions contradictoires.

Pour les initiateurs, il s'agit cependant autant d'empêcher les njuli de voir l'extérieur que de faire en sorte qu'on ne puisse pas les voir. Ce qui est sciemment recherché, c'est d'éviter à tout prix que les femmes n'entrevoient les visages des novices. Au circoncis dont le pagne glisse un peu et risquerait de laisser apparaître un peu de peau, on ordonne : «Muuri! Muuri paax!»: «Voile-toi! Voile-toi bien!» Les selbe prennent cela très à cœur et ne cessent de s'assurer que le moindre bâillement de tissu soit corrigé. Les njuli sont ainsi responsables de leur propre aveuglement. D'un interlocuteur à l'autre, on insiste plus ou moins sur le fait que ce sont les yeux ou le front que l'on doit ainsi protéger des regards féminins. Le front, dit-on, "beaucoup de gens le recherchent car cela porte chance ${ }^{41}$ \%. Mais on dit aussi que c'est au front qu'on reconnait les facultés surnaturelles de quelqu'un. Les pagnes protègeraient donc tout njuli 
doué de prescience des mauvaises intentions des gens dont on dit qu'ils ont " les yeux longs " (a ngiid a ciig). Ces êtres maléfiques que sont les naq (sorciers anthropophages) cherchent en effet prioritairement à s'attaquer aux humains qui peuvent les voir. Les jeux de dissimulation relèvent là d'une autre dimension : les pagnes permettent d'éviter une réciprocité des regards entre novices et sorciers. Les circoncis doivent donc tenir bien fermé le pagne qui les voile afin de se prémunir contre de tels risques.

Mais à l'instar de Jean Bazin on peut se demander quel est le sens profond du " dispositif de dissimulation ${ }^{42}$ " mis en place pendant cette phase du rituel. Dans un très beau texte ${ }^{43}$, il s'interroge en effet sur le protocole appliqué dans certaines cours africaines, où le visage du roi doit être caché à ses visiteurs par des effets savamment construits de voiles et d'écrans. Les situations - royauté et initiation - sont évidemment très différentes, mais leur comparaison est intéressante à plusieurs égards. Lorsque le major Dixon Denham et le capitaine Hugh Clapperton sont reçus à la cour du Bornou en 1823, le sultan apparaît doublement dissimulé à ses interlocuteurs : placé derrière un treillis de roseau, son visage est voilé de surcroît. "On voit à peine qu'il est presque totalement invisible ${ }^{44}$. "Le roi voit les autres, mais eux ne peuvent distinguer ses traits. Il s'agit d'" exhiber tout en cachant, [de] montrer sans révéler ${ }^{45}$ ". Comme le dit Octave Debary dans la présentation qu'il fait de ce texte à l'occasion de sa réédition dans L'Homme, le « corps singulier " du roi est effacé, et " cette scénographie a pour effet de produire un corps collectif $f^{46}$ ". Considérons maintenant nos njuli, dont on peut dire, ainsi que le formule Turner, qu'ils sont "structurally "invisible" though physically visible ${ }^{47}$ ". En utilisant les pagnes pour dissimuler les novices, que montre-t-on en définitive? On donne à voir des individus dont on occulte l'identité. Autrement dit, le novice n'est plus un individu, mais une représentation de l'état de njuli dans lequel il se trouve. Au visage-objet du roi tel que l'analyse Bazin, correspond le corps-objet du njuli, sans forme, encore malléable, prêt à être façonné par l'initiation. Ainsi tous les jeunes postulants au ndut deviennent, le jour de leur départ, des

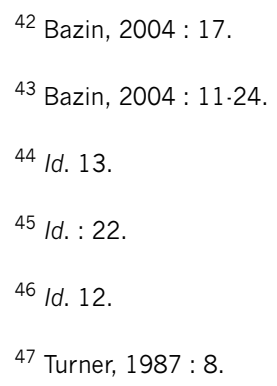


${ }^{48}$ Bazin, 2004 : 22.

49 Zempléni, 1991 :

376.

50 Bazin, 2004 : 22.

${ }^{51} / d$. : 16 njuli non seulement identiques entre eux, mais aussi identiques à tous les njuli précédents, à l'exemple du roi sans visage $^{48}$, "rappelant par-là le "caractère auto-référentiel de l'initiation" et sa "récursivité" 49 ". Pour des non-initiés, le départ des novices offre au regard une série de corps blancs anonymes, à la limite interchangeables. De sujets pensants et agissants, ils deviennent objets rituels en ce sens qu'ils sont aveugles, muets et manipulés. Objets des événements, ils sont concernés par le rite à leur insu, subissent l'action et sont d'ores et déjà les victimes qu'ils vont devenir pendant la période de marge. Ainsi les pagnes protègent. Mais ils peuvent aussi se faire carcans, bâillons et bandeaux, réduisant les novices à un état de dépendance totale.

$\mathrm{Si}$, pour les non-initiés, la personne du novice est comme dissoute sous ses pagnes, il est à noter que du point de vue des initiés, chaque novice conserve son identité propre, sa singularité. Un esclave rituel sait par exemple où se trouve son "maître " dans le cortège. Une fois dans l'enclos initiatique, les njuli, pouvant être dévoilés, retrouvent toute leur individualité. Il est en outre courant qu'on substitue à cette identité " commune " une identité " initiatique ". Lorsque les initiateurs distinguent les novices les uns des autres en leur attribuant des sobriquets ou des surnoms, ils instaurent entre eux une hiérarchie $a$ priori insoupçonnable. En effet, sous les pagnes les novices apparaissent égaux. Le tissu agit comme un uniforme au sens propre. Les pagnes égalisent les différents postulants en les dépersonnalisant, en les désincarnant. Ils permettent au rituel de produire une " image publique ${ }^{50}$ " du njuli. Au corps collectif du roi sans visage correspondrait ici le corps multiple du novice idéalisé.

Le dispositif de dissimulation du roi lui permet de voir sans être vu. L'écran, qui n'est pas opaque, donne tout loisir au souverain d'observer son interlocuteur sans être observé lui-même. Cette «non-réciprocité hiérarchique du regard [est] gênante ». Pour le visiteur, «c'est encore plus troublant que de parler à un mur ${ }^{51}$ ». Pour les njuli, la situation est différente, le rapport hiérarchique est inversé. Ce sont eux qui ne peuvent voir au-dehors et qui se savent observés. Ils reçoivent des ordres, sont chahutés, perçoivent des rumeurs, 
ne savent plus où ils sont. La gêne provient ici de l'impossibilité dans laquelle ils se trouvent de croiser les regards que les autres portent sur eux, d'y répondre éventuellement ou encore de voir qui les observent. "C'est comme si je sentais tous les yeux peser sur mon pagne " me dit un jeune initié resté anonyme, quelques jours après sa sortie du ndut. Cette situation désagréable provoque simultanément anxiété et résignation, deux sentiments que connaissent par ailleurs les mères, rappelons-le, à peu près au même moment.

Au relatif «mutisme royal " qui permet toutefois au roi de prononcer "quelques mots inaudibles ${ }^{52}$ ", pourvu qu'on ne discerne point le mouvement de ses lèvres, s'oppose l'obligation absolue de silence imposée aux novices. Ils doivent serrer les dents, et "sceller leur bouche » sous les pagnes. Un tel silence est aussi exigé des mères pendant le muur, et autrefois pendant la circoncision. Dans certains villages du Siin, on m'a rapporté du reste que le tambour martèle à ce moment-là cet ordre : "Yaay tiim, tiim! Tig a doma dom » : "Mère! Tais-toi, tais-toi! La chose est difficile (douloureuse).» Pendant la tétée symbolique que nous avons décrite, les mères serraient d'ailleurs entre leurs dents un épi de mil ou un bracelet d'argent pour s'ôter toute envie de parler à leur fils. Alors que dans d'autres sociétés le caractère hautement dramatisé de la séparation s'exprime par les pleurs ou les hurlements des mères, qui peuvent encore jeter des projectiles en direction des hommes ou s'en prendre verbalement à eux ${ }^{53}$, les mères seereer doivent souffrir en silence, comme lorsqu'elles ont donné naissance à leur enfant. Taire ses angoisses, les maîtriser, voilà qui caractérise la séparation. La douleur vient de ce que mère et enfant sont désormais sans voix, toute communication est brusquement interrompue: communication verbale, bien sûr, mais aussi visuelle. Reprenons une dernière fois la comparaison des njuli voilés au roi sans visage. Si les traits du visage royal ne sont jamais perceptibles, de même on fait en sorte que les njuli n'aient plus de visage du tout. Le roi "n'est pas censé être reconnaissable ${ }^{54}$ ", et les njuli ne doivent pas être reconnus des mères en particulier. Or des psychiatres comme Stern, pour qui « le regard est une forme

\footnotetext{
52 Id. : 22.

${ }^{53}$ Moisseeff, $1996: 31$.

${ }^{54}$ Bazin, 2004 : 19.
} 
puissante de communication sociale $e^{55}$ ", ont démontré que le lien visuel était primordial dans l'établissement du lien mère-enfant. L'attachement se nouerait dès les tout premiers contacts directs œeil-à-œil. Et c'est précisément à ce premier mode de communication non verbale auquel le pagne met un terme car il rompt ce lien visuel. Les rites de séparation visent certes à détacher l'enfant masculin de sa mère, mais afin de modifier, en le suspendant, l'attachement entre eux. Tout se passe donc comme si la séparation impliquait que l'on enclenche un processus de mé-connaissance. Une mère reconnaît son enfant partout, tout le temps; mais elle ne doit pas le reconnaitre quand il s'en va au ndut. Le pagne qui recouvre des fils provisoirement désocialisés agit de ce point de vue en soustracteur de lien.

\section{Le travail rituel des mères et le parcours des pagnes}

Quittons maintenant les rites de séparation proprement dits pour nous intéresser aux périodes pendant lesquelles mères et fils sont effectivement séparés. En amont $\mathrm{du}$ rituel, quelle est la provenance de ces pagnes qui brisent ainsi le lien maternel? Quelles obligations les mères ont-elles dans ces rites masculins? Hormis le silence auquel elles sont tenues pendant le recouvrement des novices et la reptation des circoncis, et le courage auquel on les exhorte, on attend surtout d'elles qu'elles soient généreuses. Un ndut occasionne en effet des dépenses considérables pour les proches d'un novice, et la part des mères est loin d'être négligeable.

À peine les njuli partis au ndut, les mères doivent recevoir chez elles un grand nombre de visiteurs qu'il faut combler et cela pendant au moins trois jours. Elles veillent à satisfaire tout le monde et ne cessent de distribuer de l'argent pour de menus achats : ces hôtes-ci veulent se faire du thé, ceux-là réclament de la kola, ou encore du tabac. Elles supervisent les activités culinaires dans lesquelles se lancent courageusement les esclaves rituelles des novices, quand

${ }^{55}$ Stern, 1999: $36 . \quad$ elles ne mettent pas directement la main à la pâte. Si c'est 
le père qui a pu financer les aliments de base comme le riz, l'huile, les légumes et la viande (il doit en effet tuer une vache ou un taureau pour son fils), les mères, en bonnes économes, s'assurent que les cuisinières ne manquent de rien et se chargent souvent elles-mêmes des condiments (bouillon cubes, concentré de tomates, oignons, poivre, ail, tamarin, etc.). Régulièrement, elles glissent quelques pièces à une jeune fille de la maison qui court alors «à la boutique " quérir ce qui manque. Et des sommes importantes auxquelles elles n'auraient pu avoir accès sans "l'assistance ", le soutien et la mobilisation de tout un réseau de "sœurs", filent ainsi. Leur générosité confine parfois à la prodigalité. Et quand une mère «initie " pour la première fois un enfant, les dépenses en sont d'autant plus somptuaires. Un proverbe dit d'ailleurs : " yot-yot nee woongna taaw" : "donner peu de choses ne suffit pas pour fêter la circoncision de son fils aîné56."

Toute visiteuse qui entre dans la maison s'adresse ainsi à la mère : «ndokle! » Ce salut est employé pour féliciter une personne d'avoir échappé à un danger. J'entendais d'ailleurs souvent les femmes répéter à mi-voix : "ndut naa med a med": "le ndut est lourd. "Quand on évoque la "lourdeur " du rite, on se réfère certes à son caractère dangereux, mais aussi au coût exorbitant qu'il représente en espèces sonnantes et trébuchantes comme en travail féminin. On peut encore saluer les mères en usant d'une formule qui s'apparente fort à des condoléances : mas coono fee! Les femmes y répondent par l'expression mas a las of qu'on pourrait traduire par «de même pour vous". Mas est une interjection qui exprime la compassion envers quelqu'un qui souffre; coono, c'est la douleur. Ce dernier mot revient continuellement dans les conversations féminines ces jours-là. Il ne s'agit pourtant pas, comme on pourrait le croire, de faire allusion à la souffrance que pourrait éprouver la mère séparée de son fils et de l'inquiétude qui la gagne à son sujet - que lui font-ils au ndut? - mais bien plus pragmatiquement, de la plaindre de la lourde charge laborieuse ou financière qui lui incombe. On parle aussi de fatigue (xij ou ker), notion intimement liée en seereer 
à celle de douleur ${ }^{57}$. Mais la "fatigue " ou la "douleur" des femmes dont on parle en contexte rituel tient aussi aux prestations cérémonielles et aux obligations de dons auxquelles se prêtent en premier chef les mères, mais aussi d'autres parentes des novices à la clôture de l'initiation. On retrouve d'ailleurs ce même vocabulaire dans les discussions féminines des cérémonies de mariage, où des contraintes financières comparables pèsent sur les femmes.

Parmi celles-ci, une obligation relève néanmoins de la sphère privée et de l'intimité de chaque cellule matricentrique et, à ce titre, est rarement discutée en public. Il est question de la nécessité absolue de "sortir " les pagnes des novices. Alors que le travail rituel des "pères " pendant la phase de séparation revient à manipuler les pagnes puis à en voiler les njuli, celui des mères consiste à se les procurer, puis à les céder pour un temps déterminé aux hommes et aux novices. Arrêtons-nous donc sur la provenance de ces étoffes et les manipulations dont elles font l'objet en amont du rituel, à leur sortie, puis dans le cours de l'initiation. J'avance ici l'hypothèse que ces tissus qui, comme on l'a vu, disloquent le lien maternel, lient en même temps. C'est tout le sens paradoxal que l'on pourrait donner à la succession des manipulations dont ils font l'objet : en se faisant tour à tour - et selon la lecture qu'on en fait - prolongement du corps de la mère ou du fils, les pagnes en tant qu'objets rituels portent en eux ces deux propriétés contraires. En outre, à la différence d'autres pagnes qui circulent ces jourslà, les pagnes blancs empruntent un circuit fermé dont nous verrons qu'il les ramènera finalement à leur point de départ. Nous avons détaillé deux étapes de ce circuit : le recouvrement des novices; leur départ en brousse. Nous allons maintenant évoquer les étapes antérieures.

\section{Des pagnes maternels}

Toute femme seereer se doit en principe de thésauriser, d'accumuler dans sa malle (arka) des pagnes de coton tissés qu'elle devra "sortir " (sutox) - ou «faire " $\left(f i{ }^{\prime}\right)$ - à diverses occasions cérémonielles. Dans cette zone du Hireena, c'est la mère qui est tenue de fournir les deux pagnes blancs du $n d u t$ à chacun de ses fils, mais ailleurs, une tante paternelle 
peut s'associer à elle pour fournir l'un des deux. Comment se les procure-t-elle? Plusieurs options se présentent. Le cas de figure le plus simple est qu'elle dispose d'une malle personnelle contenant des pagnes blancs (dont elle a pu, par exemple, hériter). Elle peut aussi faire valoir ses droits sur les pagnes qu'elle fait "garder " (geek) dans une malle maternelle commune que l'on appelle arka yaayaay, soit que ces pagnes lui aient été transmis, soit qu'elle les ait acquis au prix de son propre labeur. Aujourd'hui le groupe de femmes réunies autour d'une même malle se limite le plus souvent à la cellule utérine minimale regroupant des sœurs et leurs mères : ndok yaay, « case de la mère ». Dans le temps, il était plus courant que la malle fonctionne à un niveau de segmentation plus important. Selon l'importance et la localisation du segment de matrilignage concerné, la thésaurisation des pagnes s'effectuait alors au niveau du deen yaay, « sein maternel », ou du lus, « mamelon ».

Une mère provisoirement désargentée et dans l'impossibilité matérielle d'acquérir à temps les pagnes qui lui sont nécessaires alors qu'un ndut s'annonce peut aussi recourir à la réserve que représente cette malle collective, et tenter de se faire prêter les pagnes d'une autre le temps de la cérémonie. À charge pour elle alors de les restituer propres et intacts. Dans ce cas de figure, il revient à la responsable de l'ensemble des étoffes et bijoux conservés dans cette arka yaayaay - souvent la plus âgée d'un segment de matrilignage-de donner ou non son accord pour l'emprunt. Dans certaines situations extrêmes et conflictuelles, une somme d'argent (certes inférieure au coût d'un pagne neuf) peut être exigée de la requérante. Elle correspond à une sorte de droit d'emprunt, de frais de location ou encore de caution, selon la façon dont mes interlocutrices m'expliquaient cette pratique. Mais on comprendra qu'une telle solution n'est envisagée qu'en dernier recours car l'emprunteuse en serait indubitablement déshonorée, et qu'en aucun cas une mère ne voudrait s'exposer ce jour-là à l'opprobre silencieux de ses aînées. Il y a en effet de la honte à ne pas être capable de "sortir" les deux njoor d'un fils. Comme dans toute affaire de pagnes, la réputation de la pourvoyeuse est en jeu. Il peut arriver néanmoins qu'une détentrice de pagnes 
généreuse en prête ou en avance spontanément et sans contrepartie immédiate à une mère dans le désarroi. Ce geste demeure alors très discret, de sorte qu'il nuit rarement à la réputation de la destinataire du prêt.

Le plus souvent donc, les femmes ont tout intérêt à anticiper leurs besoins, afin de faire face à l'éventualité toujours probable qu'une occasion de "sortie " de pagnes se présente. Pour un ndut, et dès qu'elles ont un peu épargné, elles se mettent en quête de ces tissus. Lorsqu'elles s'y prennent vraiment à l'avance, elles peuvent obtenir du coton local (souvent contre services rendus) que des vieilles de la famille auront passé de longues heures à égrener et filer à la main. Rares sont en effet aujourd'hui les jeunes femmes qui cultivent et transforment artisanalement le coton. Elles peuvent aussi, en complément ou pour la totalité du pagne, se procurer directement du fil industriel auprès d'un marchand. Un pagne blanc est d'ailleurs souvent tissé des deux fils : artisanal pour la trame, industriel pour la chaîne. Quel qu'il soit, ce fil sera en fin de compte confié aux soins d'un tisserand, griot seereer ou tisserand tukulëer installé dans les environs pendant la saison sèche. Le tissage est souvent rétribué en espèces pour une part et en nature pour une autre. De fait, durant tout le temps que le tisserand passe sur le pagne de sa cliente, elle est censée lui fournir thé, tabac, repas, et parfois même logement. Une mère peut enfin - et c'est plus rare - acheter directement des pagnes finis ou plus couramment des rouleaux de bandes ou lés de coton déjà tissé. Il ne lui restera plus qu'à coudre bord à bord 7 bandes (d'environ $20 \mathrm{~cm}$ de large sur 1,20 m de long) pour obtenir le njoor voulu. La plupart de mes interlocutrices s'accordent sur ce point: plus la démarche d'acquisition est longue, plus le pagne obtenu porte l'empreinte d'un travail de la mère - lequel porte en soi la trace de celui d'une ou de plusieurs aînées lorsque le coton a été filé à la main -, et plus le pagne sera valorisé et la mère estimée. Par contraste, la brièveté d'une transaction marchande qui permettrait d'obtenir un pagne achevé prive le geste maternel de toute profondeur, ôte du sens à la sortie publique de l'objet. Autrement dit, plus la mère 
y met de la peine, plus «lourd" (medu) et donc plus beau sera le pagne. Cette conception qui associe la belle facture et les qualités d'une étoffe au labeur de la mère trouve son origine, semble-t-il, dans l'obligation que toute mère avait dans l'ancienne société seereer, de cultiver, égrener, carder, filer, faire tisser, puis coudre enfin les vêtements de toute sa famille. La beauté ou la valeur intrinsèque de l'objet ne réside ici ni dans des élaborations esthétiques déterminées, ni dans le parcours qu'il a pu effectuer (comme un objet kula par exemple) mais bien dans l'empreinte invisible que laisse au plus profond de ses fibres la sueur de la fileuse ou de la couturière. L'image d'une mère épuisée par ses multiples travaux diurnes et qui, au mépris de la fatigue, s'abîme de surcroît les yeux à la faible lueur d'une lampe à huile sur son ouvrage est très présente à l'esprit des hommes comme des femmes les plus âgées. C'est le modèle de la mère courageuse. D'ailleurs, ne dit-on pas : «njambaar o lalta yaa' fa jabu": "c'est le courage qui montre la bonté d'une mère ${ }^{58} "$ ?

Mais il arrive que des mères se trouvent dans l'impossibilité de fournir à temps les pagnes des novices. Il en est ainsi, par exemple, lorsque des garçons fuient leur village d'origine pour se faire initier ailleurs contre la volonté de leurs parents ou à leur insu. Les initiateurs, lorsqu'ils ont décidé de les accepter, chargent alors les plus proches parents ou simplement des personnes de même matriclan de désigner la femme qui sur place, devra fournir les pagnes nécessaires à leur initiation. Un jeune garçon avait ainsi fui Djilor pour se faire initier. Quand sa fugue fut découverte et sa destination connue, la grand-mère maternelle du petit fugueur fit prévenir sa sœur cadette mariée à Yayème et c'est à cette dernière qu'échut le devoir de retirer de sa malle les deux pagnes de son petit-fils classificatoire.

Outre les njoor, une mère se doit également d'offrir un pagne noir (pay baal) - bleu en fait car teint à l'indigo ou un pagne rayé de lignes noires et blanches pour confectionner une chemise (forok, du français «froc »). Ces pagnes à rayures sont très rarement nommés par les femmes: on emploie surtout les qualificatifs de « joli " (pay mossu), ${ }^{58}$ Crétois, 1972.1977. 
" tissé " (pay tiwand) ou " traditionnel " (pay coosaan) pour les désigner. Le nouvel initié enfilera son forok par-dessus les habits neufs que son père lui aura achetés pour assister aux danses des esclaves et aux échanges de dons qui clôtureront l'ensemble du rituel. En fait de chemise, c'est simplement un pagne au centre duquel on ménage une ouverture, dans la longueur, afin d'y passer la tête. Les pans de tissus retombent alors sur les épaules du jeune homme, un peu à la manière d'une cape ou d'un poncho. À défaut de pagne "traditionnel ", un pagne dont le tissage emploie des fils de couleurs vives (teintures chimiques) et brillants (lurex) et qu'on attribue aux Tukulëër, Wolof ou Njaago (Manjak), fera l'affaire. Ainsi le dit cette complainte :

Njegangee pay Gaal, ñaapik Gojang ee, Buure Sambu Jeegaan,

Si je n'ai pas de pagne noir, je chercherai du bojang, Bouré Sambou Diégane,

ke fuma woloor no cuul ee

pour mettre en valeur mon circoncis.

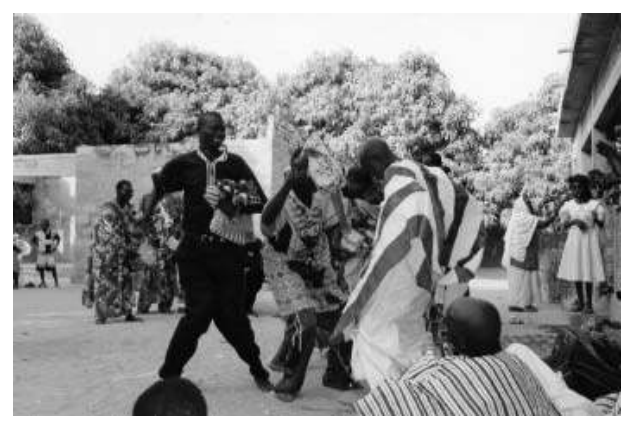

8. Danses des esclaves rituels qui viennent de recevoir pagnes et tissus devant les nouveaux initiés en forok (Yayème, 2000)

Dans ce chant, une mère se lamente de ne pas avoir encore trouvé, à quelques semaines d'une initiation, de pagne noir pour confectionner le forok de sortie du ndut de son fils. Elle se console en se disant qu'elle trouvera bien du bojang, ce fil de lurex doré ou argenté qui entre de nos jours dans la fabrication des pagnes que l'on appelle rabal awo en wolof, littéralement "pagne tissé de première épouse ". Enfin, elles doivent faire tisser un autre pagne noir ou joli pagne par esclave rituel masculin, en rétribution du rôle qu'il a tenu auprès du njuli pendant le rituel. 
Afin d'illustrer ces considérations générales, je propose d'examiner maintenant l'exemple de Yacine, dont les apports de pagnes ont été schématisés dans la figure 2 ci-dessous. Yacine est la mère réelle (yaay no fud, "mère de ventre ") de trois garçons, et la mère adoptive du fils que son mari a eu d'un premier mariage rompu. Tous les quatre se sont fait initier en 2000. Respectivement nés en 1975, 1979, 1982, 1986, tous se sont fait circoncire très jeunes au dispensaire.

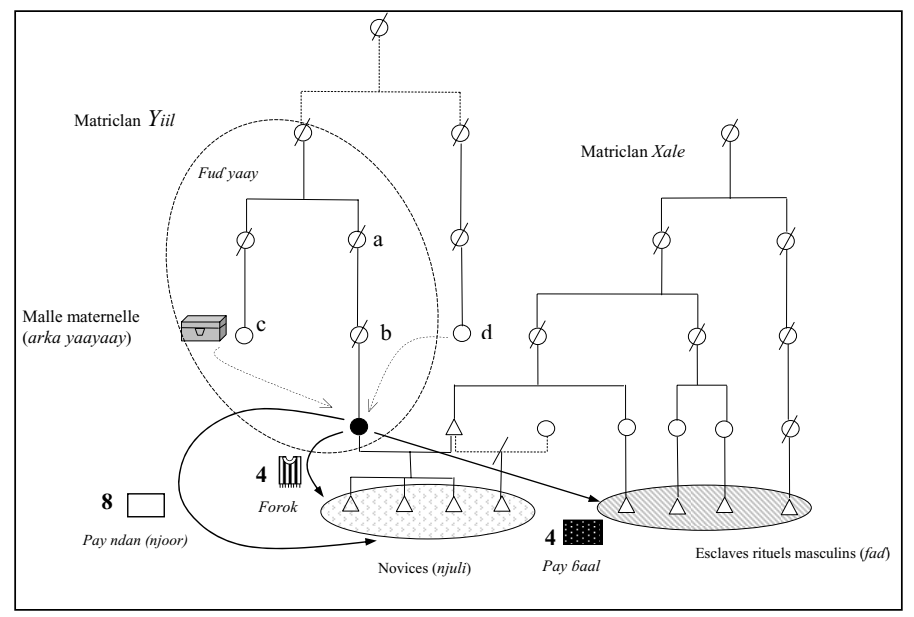

Figure 2 - Les obligations d'une mère : les 16 pagnes de Yacine Diop (Yayème, 2000)

Les pagnes blancs (njoor) qu'elle dut sortir pour leur ndut proviennent d'une malle conservée chez sa ya fa ndeb, "petite mère " (c) - une sœur cadette et classificatoire de sa mère décédée. C'était sa grand-mère maternelle (a) qui les avait fait tisser. À son décès, la mère de Yacine (b) en hérita. Lorsqu'elle mourut à son tour, sa malle et une bonne part des pagnes qu' elle contenait fut confiée à (c), qui restait alors la plus âgée des femmes du fud yaay ou " ventre de la mère ", segment de matrilignage minimal englobant plusieurs ndok yaay (case de la mère) d'origine commune. Celle-ci se trouvait donc en 2000 à la tête d'une arka yaayaay conséquente dans laquelle venaient régulièrement puiser plus d'une dizaine d'ayant droit: autres "mères" de Yacine, sœurs et cousines parallèles matrilatérales. Bien que Yacine fût 
59 Boser-Sarivaxénavis, $1969: 153 \cdot 161$. trop jeune encore pour avoir le droit de les conserver dans sa propre maison, les huit pagnes blancs qu'elle alla retirer pour les quatre njuli étaient les siens propres, puisqu'elle en avait hérité personnellement de sa mère. Tenue de les rapporter chez (c) à la fin du rituel, elle dut en outre payer un bloc de savon neuf pour les laver. Ce n'est que lorsqu'ils furent propres, repassés, pliés avec soin, puis emballés dans de vieux foulards, qu'elle s'en alla les remettre à (c) qui les rangea dans sa malle. De cette manière, ils étaient rendus de nouveau disponibles pour un événement ultérieur.

En ce qui concerne les pay baal (pagnes noirs) des esclaves rituels de ses fils (dont nous voyons sur la figure 2 comment ils sont apparentés un à un avec les njuli), elle s'y était prise dès le mois de novembre 1999, pour le mois d'avril 2000, alors que les premières rumeurs qu'un ndut pourrait bien avoir lieu se faisaient entendre. Elle acheta alors des bandes de coton blanches déjà tissées pour l'équivalent de quatre pagnes à des marchands tukulëër de Diourbel pour 10000 francs CFA l'ensemble (soit environ $15 €)$. Après avoir assemblé elle-même les pagnes, elle les confia à une teinturière seereer de sa connaissance, installée à Dioffior, qui lui demanda 6000 francs CFA pour ses services (soit environ $10 €$ supplémentaires). Ce prix comprenait les différents travaux de préparation du tissu dont se charge alors la teinturière : ligatures et fronces - afin d'obtenir les réserves cordées typiques de "l'École du Sénégal ${ }^{59}$ ", préparation des différents bains de teinture, retrait des fils et repassage des pagnes secs. Un total de 16000 francs CFA, sans compter le coût du transport, représentait une somme importante. Yacine disposait de petits revenus générés par son travail à la case de santé du village. Son époux, bien qu'il fût salarié, n'était pas impliqué dans ces dépenses. Un ami de Yayème me dit un jour : "Ça ne nous regarde pas, nous les maris : ce sont des affaires de femmes (l'acquisition de pagnes). "Quant aux pagnes rayés qui servirent de forok, ce fut une autre de ses mères classificatoires (d) qui les apporta et les lui remit quelques semaines avant le ndut. Ces pagnes, Yacine les donna pourtant en son nom propre pour la sortie. Car ici, comme pour les pagnes que reçoivent les 
$f a d$, on ne parle plus de "faire " $\left(f i^{\prime}\right)$ un pagne, mais bien de le « donner " (ci', cood ou encore coox); il s'agit d'un présent. Chaque njuli, comme chaque fad d'ailleurs, gardera ensuite son forok (ou son pay baal) et en disposera comme bon lui semble. Il pourra le porter à d'autres occasions festives, le faire coudre comme chemise à l'occidentale, voire le reconvertir en simple couverture.

Outre les pagnes tissés, d'autres tissus furent rassemblés en masse dans une chambre de la maison de Yacine, dans laquelle des parentes utérines et agnatiques des njuli s'enfermèrent au lendemain du départ des novices afin de procéder au partage des différents coupons que des donatrices de provenances variées accumulèrent sur un lit (photo 9). En présence de la mère des njuli et sous l'égide d'une ou deux aînées dont la personnalité et l'autorité s'affirment en de telles circonstances, elles passèrent en effet un temps considérable à trier, palper, admirer ou critiquer, compter et mesurer, répartir, attribuer et enfin organiser par lots ces tissus colorés.

Sont habituellement exclues de ces réunions les jeunes femmes non ou trop récemment initiées. De fait la moyenne d'âge y est relativement élevée, la mère des njuli étant certainement la plus jeune de toute l'assemblée. Voisines ou résidentes d'autres villages dont l'éloignement les fait rares à Yayème, toutes ces dames prennent un plaisir certain à se retrouver ainsi entre elles. Le très grand sérieux avec lequel elles considèrent ces affaires de partage, et qui les entraîne sur les sentiers souvent épineux de la parenté et de la bienséance, ne les prive pas de distractions. Plaisanteries, chants, et parfois un ou deux pas de danse, égayent et entrecoupent ce temps de la répartition. Les plus gros lots de tissus ainsi accumulés sont apportés par des tantes paternelles des njuli ou en tout cas des femmes du matrilignage de leurs pères. Il est alors intéressant de remarquer que parmi ces pièces de tissus, nombreuses sont celles qui en définitive seront récupérées par leurs propres enfants, puisque ce sont précisément parmi eux que sont recrutés les esclaves rituels des novices. Ces cadeaux, qui n'ont d'existence que parce que le déroulement d'un ndut leur donne une occasion de se 
réaliser, revêtent donc potentiellement la forme de dons indirects de mères à leurs enfants. On pourrait avancer l'hypothèse qu'ils "répondent", en le déclinant sur un autre mode, au don direct du forok de la mère du njuli et à l'inverse "s'opposent " ou " concurrencent " le don de pagne tissé que cette dernière fait aux esclaves rituels de ses fils.

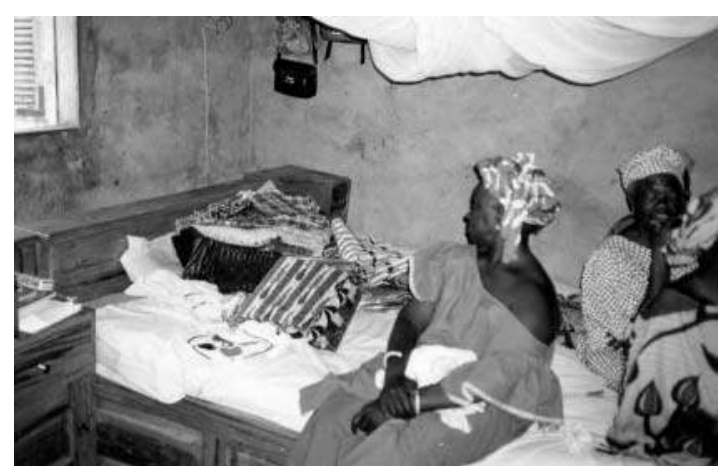

9. Tissus destinés aux dons de la sortie du ndut et aux esclaves rituels (Yayème, 2000 et 2001)

Destinés à être distribués à la clôture du ndut, lorsque les nouveaux initiés vêtus de leur forok apparaîtront en public assis sur une natte et dévoilés pour la première fois, tous ces tissus vont être exhibés. Leur distribution donne lieu à des danses. Lorsqu'ils sont appelés, les esclaves masculins et féminins des initiés reçoivent leur lot des mains des femmes qui ont pris part au partage. Ils vont alors manifester leur joie en allant danser devant leurs " maîtres". Les billets que des spectateurs séduits par leur prestation leur remettent sont accrochés à leur poitrine au moyen d'épingles à nourrices ou directement fourrés dans leurs bouches. Certains se ceignent simplement la taille des tissus ou les portent à l'épaule.

D'autres plus hardis exécutent des danses suggestives dont les coups de reins envoient voler à la verticale les pans de tissus, suggérant d'énormes et fugaces érections qui arrachent des fous rires à l'assistance. L'élément comique est renforcé par les inversions auxquelles se livrent parfois ces danseurs en se parant des attributs vestimentaires de l'autre 
sexe. Le tissu reçu, ainsi que le costume choisi, deviennent des éléments autour desquels chacun à sa guise et selon son audace construit sa danse.

Ces cadeaux que reçoivent si joyeusement les fad représentent la juste rémunération de leur service d'esclave rituel. Ainsi les femmes sont publiquement remerciées pour leur cuisine; les garçons pour le rôle de "valets " des njuli qu'ils ont tenu au ndut (transport des repas, des pagnes, protection de leur " maître " contre les coups trop rudes des selbe, etc.); tous pour leurs danses. Ces dons sont étendus à tous les cousins croisés patrilatéraux des njuli, et à tous ceux qui, en tant que " fils » ou « fille » du matrilignage de ce dernier, sont présents ce jour-là et estiment être en droit de venir «s'engraisser".

Pour reprendre mon exemple précédent, chez Yacine, chaque esclave masculin a reçu en plus de son pagne noir, 6 mètres de tissu leegos ${ }^{60}, 10$ mètres de tissu xaartum ${ }^{61}$, un foulard de tête et 2500 francs CFA (4€). Chaque esclave féminine a reçu 12 yards de tissu dit dampi ${ }^{62}, 6$ mètres de xaartum, une camisole et 530 francs CFA (soit un peu moins d'un euro). Avec quatre garçons à initier, la charge pour Yacine, simplement en termes de textiles, est lourde. Mais j'ai rencontré des femmes qui non seulement devaient fournir des pagnes blancs pour leurs fils mais aussi pour des enfants de sœur. Ainsi l'exemple de Fatou Basse qui, heureusement soutenue par sa mère et grâce à la mise en commun de pagnes utérins, put fournir en 2001 pour six njuli orphelins de mère, vingt-quatre pagnes en tout - douze pagnes blancs, six pagnes noirs pour les esclaves rituels et six pagnes noirs de plus pour les habits de sortie.

Ces pagnes que les mères ont donc plus ou moins de peine à rassembler sont des objets de l'ombre et de l'intimité, tant qu'ils ne font pas l'objet de cette "exposition révélatoire ${ }^{63}$ " qu'est leur entrée en scène publique sur le corps des novices. Enfermés dans des malles, souvent stockées sous les lits, dans le secret des chambres, c'est dans une grande discrétion qu'ils sont portés aux maris de ces mères, avant que ces derniers ne les confient ensuite à qui voilera

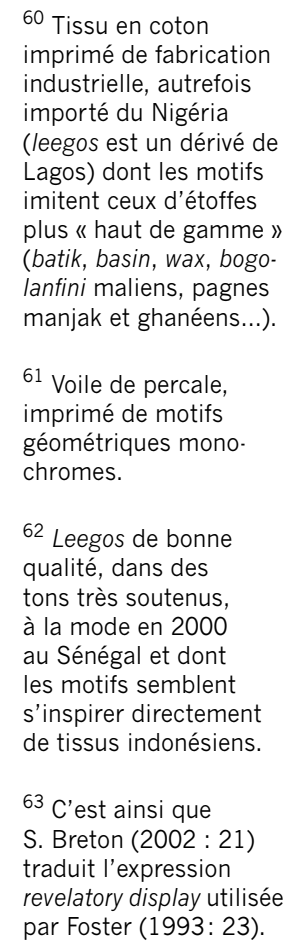

61 Voile de percale, imprimé de motifs géométriques mono. chromes.
62 Leegos de bonne qualité, dans des tons très soutenus, à la mode en 2000 au Sénégal et dont les motifs semblent s'inspirer directement de tissus indonésiens.
${ }^{63}$ C'est ainsi que S. Breton (2002 : 21) traduit I'expression revelatory display utilisée par Foster (1993: 23).




\footnotetext{
${ }^{64}$ Ndiaye, 1986 : 34 35; Gravrand, 1990 : $47.48 ; 52.53$

65 Dione, 2004 : 24.

${ }^{66}$ La Fontaine, 1985 : 120.
}

les njuli (un "père " puis un esclave rituel). Cette "passation" des pagnes blancs se fait toujours à l'aube, dans la pénombre, par ballots discrets, après qu'ils ont été comptés et recomptés, attribués nommément à chaque circoncis par la mère et l'une de ses filles ou nièces, et enveloppés dans des foulards de tête.

Autrefois, les mères, des sœurs ou des tantes paternelles selon les régions, offraient encore les vêtements ou parures (coiffes, colliers, cauris, bracelets) que portaient les incirconcis lors des danses préliminaires à la circoncision. Des baudriers faits de bandes de cotonnade pouvaient être agrémentés de miroirs, de perles ou de coquillages. Pour l'occasion, les jeunes garçons s'étaient laissé pousser les cheveux, qu'ils se faisaient ensuite tresser. C'est avec cette allure plus féminine que masculine qu'ils se présentaient devant le yaaman. On appelait alors l'habit du novice ndoki jambar, "habit du courage ${ }^{64}$ ". Exactement une semaine avant la circoncision, dans le Siin, la tante paternelle du jeune postulant passait un bracelet d'argent au poignet gauche de son neveu qui avait pour rôle "de sceller sa décision inéluctable d'entrer dans la société des hommes par le biais de la circoncision ${ }^{65}$ ». Enfin, notons encore qu'à Simal, village voisin de quelques kilomètres (voir carte 2 ), il est de coutume d'agrémenter le forok de sortie des nouveaux initiés de colliers ou de chaînes avec de gros pendentifs. Ils peuvent être prêtés par les mères ou donnés par les sœurs des novices, ou une de leurs amies. Dans son ouvrage comparatif sur les institutions initiatiques, Jean La Fontaine ${ }^{66}$ note qu'il est courant qu'un postulant à l'initiation masculine doive porter des éléments de parure féminins : il s'agit selon elle de l'identifier à la parentèle féminine et de le faire bénéficier de la force que conférerait le pouvoir régénérateur du lignage. Dans le cas seereer, j'invoquerais simplement la capacité qu'ont ces objets divers à témoigner des relations que peuvent avoir les njuli avec leur entourage féminin. Les bijoux (colliers de cornaline ou en perles de Venise, bracelets d'argent) qui, lorsqu'ils ne sont pas portés, côtoient d'ailleurs les pagnes dans les malles, révèlent les liens qui unissent un frère et une sœur, des amis de sexe 
différents. Le bijou, un peu à la manière du pagne, imprime sur le corps du novice l'empreinte de la femme ou de la jeune fille qui l'offre ou le fournit.

\section{Un travail nocturne}

L'examen d'un épisode singulier du parcours des njoor, alors que les novices sont en brousse, permet d'éclairer la manière dont le rituel joue sur le lien maternel. Au premier soir du ndut, à la tombée de la nuit, les esclaves rituels rapportent au village en toute discrétion les pagnes blancs que les novices ont portés toute la matinée pendant le recouvrement et la reptation des circoncis. Leurs mères sont alors tenues d'utiliser la pile de ces pagnes pliés en guise d'oreiller. Elles passeront ainsi chaque nuit que les fils sont au ndut, la tête posée sur les étoffes sous lesquelles, quelques heures plus tôt, ils étaient dissimulés. Puis, à l'aube de leur retour au village, les pagnes empaquetés seront rapportés par les esclaves rituels au camp de brousse. Les garçons nouvellement initiés en auront alors une dernière fois l'usage lorsqu'ils regagneront le village au crépuscule et iront "saluer" par petits groupes le lendemain matin, avant de revêtir enfin leurs tuniques de sortie (forok) et de consommer le mupter, un repas offert par les mères et qui les autorise à « ouvrir la bouche » et à s'adresser à elles de nouveau.

Cette coutume des pagnes-oreillers de Yayème semble aussi connue à Djilor Djidjak (village "souche " à partir duquel Yayème a été fondé dans les années 1880). Je ne peux cependant pas m'avancer quant à son existence ailleurs, mais il semble bien que le fait que les pagnes blancs soient rendus aux mères le temps de la retraite en brousse soit actuellement une pratique courante dans la zone d'enquête. En revanche, pour ce qui concerne une époque révolue, celle qui voyait les ndut durer de longues semaines, mes interlocuteurs les plus âgés ne s'accordent pas quant à l'usage que l'on faisait des pagnes du muur pendant la retraite. Ils servaient semble-t-il à voiler les novices chaque fois qu'ils s'approchaient du village ou risquaient d'être surpris par des non-initiés, mais les souvenirs sont 
imprécis quant au fait qu'on les restituait ponctuellement aux mères ou non.

Dans d'autres contextes comme ceux des rites funéraires, des pagnes blancs peuvent également être employés à la manière d'oreillers. C'est le cas chez les Seereer catholiques, qui continuent d'inhumer leurs morts enveloppés et accompagnés de nombreuses étoffes. À Fadiouth par exemple, tout défunt "part " avec, au minimum, trois pagnes blancs. Son corps repose sur le "pagne de père ", étendu au fond du cercueil, alors que le "pagne des maternels " le couvre. C'est le troisième pagne, apporté par ses beaux-parents, la veuve ou le veuf qu'il laisse ou encore par les affins de ses fils, qui est roulé ou plié sous sa tête et sert d'oreiller. Il est aussi fréquent de voir la tête du nouveau-né assoupi reposer sur un pagne blanc plié que sa mère ou sa grand-mère maternelle a fait tisser pour lui. Mais à ma connaissance, ces deux autres exemples de pagnes-oreillers ne font pas l'objet de discours aussi élaborés, ne sont pas investis d'autant de significations que ce qu'il m'a été donné d'entendre à Yayème dans le contexte initiatique.

Deux interprétations sur la fonction de cette pratique sont en effet avancées par les femmes qui en ont fait l'expérience. Elles sont divergentes. La première suggère que la mère, en dormant sur les pagnes de son fils, protège à distance son enfant: en rêvant, elle lui transmettrait de «bonnes pensées » censées le réconforter et le rassurer. Les tissus serviraient ainsi de "vecteurs " aux pensées maternelles, de moyen d'expression de secours (puisque les moyens de communication ordinaires lui font défaut). Ils lui permettent en somme de rester mère malgré l'absence du fils, de conserver son rôle de protection et de dévouement. Elle pourrait ainsi, dit-on, s'assurer qu'un enfant dont elle connait les qualités, et qui ne mérite aucun traitement sévère, ne soit pris pour un vaurien par les selbe et battu en conséquence. Ici, et comme le remarque Turner, "mothers and novices are mystically connected during seclusion ${ }^{67}$ ", à la différence près que dans l'exemple ndembu qu'il cite, cette

${ }^{67}$ Turner, 1962:159. « connexion » se fait au moyen d'un chant. 
La deuxième raison invoquée pour éclairer cet usage semble dire tout le contraire. En fait de bonté et de souci de sa progéniture, la mère serait potentiellement malveillante à son égard. Le pagne qui, après avoir rompu le lien visuel mère/fils, fait obstacle aux regards de personnes hostiles, empêche de la même manière la mère d'atteindre son enfant par les voies oniriques. Il devient ici une "barrière" à pensées, ou encore un "piège " à rêves qui protégerait ses enfants de sa propre inclination, que d'aucuns jugent " naturelle", à être une " mère dévorante " ${ }^{68}$. Sans les pagnes qui bloquent ses pensées nocives et retiennent son double sorcier dans l'enceinte du village, elle risquerait d'aller rôder près du ndut. Dans cette seconde interprétation - qui est, avouons-le, la seule avancée par des hommes lorsqu'ils en parlent -, la mère est donc perçue comme pouvant à tout instant mettre en péril l'initiation de son fils, aussi éloigné et séparé d'elle soit-il. Ce qui s'exprime ici ne se résumerait-il pas à cette simple affirmation, qu'une mère, de toute façon, représente une menace du point de vue de la nécessaire transformation de son fils en homme?

Dans ces deux cas de figure, par cet acte nocturne, la mère protégerait donc son fils, soit des mauvaises dispositions des autres (les selbe) à son égard, soit de sa propre propension à lui être nuisible. Elle se protégerait elle-même de surcroît. Qu'on lui prête de bonnes ou de mauvaises intentions, les pagnes qui la détournent de l'enclos initiatique où elle pourrait nuitamment tenter d'apercevoir ou d'anéantir son fils, la préservent elle-même. Ils semblent là pour brider ses ardeurs maternelles et la détourner du danger de mort qu'elle court. Puissants contre-sorciers, le kumax ou le kalma qui la verraient (en rêve eux aussi) s'approcher ainsi de l'enceinte du camp réagiraient à la première alerte et n'hésiteraient pas à la tuer sur-le-champ. Quoique d'apparences contradictoires, puisque d'un côté les pagnes réunissent les personnes séparées et de l'autre ils renforcent la séparation déjà effective dans la réalité en la rendant efficace aussi dans le monde nocturne, ces deux interprétations sont étroitement imbriquées dans les discours et semblent nécessairement complémentaires. Du 
${ }^{69}$ Schneider \& Weiner, 1986 : 178.

70 Latry, 1996. reste, les femmes interrogées à plusieurs reprises à ce propos donnaient indifféremment, successivement ou conjointement l'une ou l'autre de ces explications. Qu'en ressort-il? Plusieurs hypothèses peuvent maintenant être avancées quant à quelques propriétés remarquables des objets qui nous intéressent ici.

Les pagnes ont la capacité de porter en substance l'essence, les traces des personnes qui les ont manipulés, touchés, portés. Le tissu couvre, enveloppe ou habille le corps et, comme le soulignent Schneider et Weiner, «its intimate association with the body is particularly salient, putting it in a metonymic relationship to the self $f^{69}$ ". Par sa capacité à avoir conservé en sa trame les traces de la mère qui l'a "fait " et du fils qui en a été voilé, il met deux personnes en relation. Grâce à ce travail nocturne (dormir sur les pagnes), mère et fils que le rituel s'efforce de séparer sont ainsi mis en contact. En outre, dans le cas que l'on examine ici, le textile est support de rêve. Il n'est pas rare de voir ainsi rapprochées activités oniriques et matières tissées ou activités textiles féminines. Ainsi, Marie-Claire Latry décrit comment le rêve agissait dans l'atelier de couture du Bordelais pour réconcilier des personnes et établir " une passerelle [...], un pont entre vivants et morts, cordage jeté entre leurs mondes respectifs ${ }^{70}$ ". De même, l'activité onirique de la mère et les pagnes blancs jettent cette passerelle entre village et brousse, entre case maternelle - fondamentalement importante, ne l'oublions pas, dans la société seereer, et constitutive de l'identité et de la personne - et le "nid" (ndut) des hommes. Si ici le rêve n'a pas à "réconcilier " mère et fils (comme c'est le cas dans l'atelier de couture bordelais), on lui reconnaît la capacité à les réunir.

Quelle que soit donc la fonction que l'on prête à la pratique du pagne-oreiller (barrière/piège, ou moyen de protection à distance) les pagnes évoquent aux mères leurs fils, sont porteurs de leur empreinte, et sont en définitive le signe d'une relation qui perdure et que maintient le rituel malgré ses intentions affichées. Cette pratique témoigne du caractère indéfectible du lien maternel et instaure une discontinuité dans le processus de séparation : l'espace d'une 
nuit, la mère peut avoir le sentiment d'être proche de son fils. Cet acte renvoie indéniablement à la relation infantile de la mère à son fils et peut se lire comme un retour sur la petite enfance de ce dernier. On sait que dans la société seereer comme dans la société wolof, le petit enfant dispose "librement et pendant longtemps du corps de sa mère ${ }^{71}$ ". Les pagnes s'immiscent très tôt dans cette relation : ils sont employés dès la naissance. Ils se font langes, draps, couvertures. La mère et son bébé dorment ensemble dessus. Ils lient étroitement leurs corps dans le portage tout le temps que dure l'allaitement maternel, et parfois même un peu au-delà (soit 18 à 24 mois en moyenne). Pour les quelques mères qui évoquent le sentiment que leur inspirait cette pratique, ou pour celles qui cherchaient à m'en faire comprendre la portée affective, ce "travail nocturne " est comme une dernière berceuse (a kim a yaal) pour leur fils. On voit, comme l'observait Juillerat à propos du naven des Iatmul, que «le vécu de l'enfant et de la mère [...] devient un matériau repensé, manipulé, transformé [...]», que «la séparation d'avec elle n'est jamais radicale ${ }^{72}$ " car «le lien de l'enfant à sa mère est fondé sur une relation corporelle et affective forte, alors que la relation au père a un caractère distant et prospectif ${ }^{33}$ ". Le rite de la dernière tétée décrit plus haut relève de cette même logique rituelle qui met en œuvre plusieurs effets jouant sur le lien maternel.

Mais s'il semble réunir les deux êtres séparés, cet usage particulier des pagnes signifie tout aussi cruellement l'absence du fils. Les mères dorment sur ces pagnes qu'elles ne considèrent plus être les leurs (bien qu'elles les possèdent en propre, qu'elles les aient fournis) mais bien comme étant ceux de leurs fils absents. On peut alors considérer que le tissu permet à la mère de passer ce cap de la séparation, de l'aider à accepter la situation, et de l'apaiser. Dans ce contexte particulier, les pagnes feraient office de véritables objets transitionnels, mais dans un sens légèrement détourné pour les besoins de l'analyse et qui se rapprocherait un peu de ce que Droogers ou Turner ${ }^{74}$ entendent lorsqu' ils parlent de «symboles transitionnels ». Pour la psychanalyse, l'objet transitionnel apparaît lorsque l'enfant construit la sépara-

\footnotetext{
${ }^{71}$ Rabain, 1994 : 90

72 Juillerat, 1999 : 157.

${ }^{73} / d .: 173$.

${ }^{74}$ Droogers, 1980; Turner, 1967.
} 
${ }^{75}$ Winnicot, $1975: 50$.

76 Juillerat, $1999: 156$.

77 Juillerat cite notamment Winnicot (1975).

${ }^{78}$ Stern, $1999: 338$. tion entre sa mère et lui. Il est souvent qualifié de première possession en propre représentant ce dont il est en train de se séparer, c'est-à-dire sa mère. Il est très courant dans nos sociétés qu'un objet textile (morceau de couverture, foulard, poupée de chiffon) soit cet objet transitionnel. Pour le petit enfant seereer, on peut observer au quotidien le rôle rassurant que peut jouer le pagne de portage : lorsque le bébé est descendu du dos maternel, il est posé ou couché sur les tissus qui ont servi à le porter. Il peut à loisir les mordiller, les caresser ou les palper au même titre que certaines des amulettes dont son corps ou le corps de la mère est orné. Quand la mère est absente, l'objet transitionnel donne l'illusion rassurante de sa présence et sert à calmer ses angoisses. Il aide mère et enfant à se détacher l'un de l'autre. Pour Winnicot, cet objet « représente la transition du petit enfant qui est en passe de l'état d'union avec la mère à l'état où il est en relation avec elle, en tant que quelque chose d'extérieur et de séparé ${ }^{75}$ ". Lorsque la mère seereer dort sur les pagnes de son fils reclus au ndut, à l'inverse, c'est le fils absent qui demeure symboliquement présent pour la mère; ses pagnes sont un prolongement du fils et la mère peut les investir à ce moment comme tels.

Par ailleurs, et Bernard Juillerat l'a admirablement démontré dans le cas du naven des Iatmul, la séparation d'avec la mère ne peut s'effectuer sans retours vers elle : "Au-delà de la nécessité de la séparation, il y aura permanence du lien, une constance affective ponctuée de "retours" réels ou symboliques ${ }^{76}$ ". Son analyse s'appuie en partie sur les travaux de théoriciens de l'attachement ${ }^{77}$, pour qui le parcours du petit enfant vers l'autonomie est jalonné de ces retours successifs à l'occasion desquels il peut se "ravitailler » et, ainsi que le formule Daniel N. Stern, " prendre quelque chose qui lui permette de repartir et de reprendre son exploration [...], de se séparer à nouveau et de repartir explorer ${ }^{78}$ ». Ces recherches montrent comment l'enfant est celui qui prend l'initiative de l'éloignement comme de ses retours. Par le «non! » qu'il acquiert peu à peu, il choisit les moments où il ne souhaite plus être en rapport avec sa mère (en s'éloignant un peu et en détournant son regard, par 
exemple), avant de revenir vers elle se rassurer et prendre la mesure de l'expérience d'éloignement et d'autonomie qu'il vient de faire. En outre, pour Stern, ces retours ont des buts multiples qui permettent « un partage subjectif - le réétablissement d'un état d'intersubjectivité, qui n'est pas un état donné, mais qui doit être maintenu ${ }^{79}$ ". Or l'examen de la phase de séparation du rite d'initiation masculine seereer - comprise encore une fois, comme les rites qui marquent le départ des novices vers le camp de brousse et le travail rituel de leurs mères alors qu'ils sont absents du village - révèle qu'on se trouve encore une fois ici dans une situation inversée par rapport à la petite enfance. Dans le ndut seereer, ce n'est pas au fils que revient la responsabilité des retours vers la mère. Au contraire, et si l'on se place du point de vue des initiateurs, tout indique que c'est toujours la mère que l'on soupçonne de vouloir approcher son fils, d'être tentée de le rattraper, bref de vouloir faire retour vers lui. Elle risquerait à tout instant de le reconnaitre en cherchant à le voir, dans une tentative parfois désespérée de faire durer, encore un peu, les échos de la petite enfance qui planaient dans leur relation. Le rituel la place alors dans des situations où elle est contrainte d'agir dans le sens des actions des initiateurs et donc empêchée d'agir selon son propre désir. C'est le cas dans les différentes étapes qui ont été décrites plus haut : c'est la mère, "complice " des initiateurs, qui offre son sein au garçon lorsque la dernière tétée a lieu; c'est elle qui fournit les pagnes mais ce sont les initiateurs qui en font usage pour rompre le lien visuel en interdisant toute réciprocité de regard entre eux. Enfin, et dans le cas que l'on vient d'examiner, quand la mère dort sur les pagnes de son fils, c'est elle que l'on soupçonne de tenter de le rejoindre. Ainsi le travail rituel de la mère peut faire l'objet d'une double lecture, ses actes étant à la fois dirigés vers son fils et contre elle-même.

De nombreux travaux sur l'initiation masculine ont mis l'accent sur la période de marge ou de réclusion des novices. Turner ${ }^{80}$ en particulier, fut parmi les premiers à souligner l'importance de la phase liminale pendant

79 Ibid. : 341.

80 Turner, 1987. 
${ }^{81}$ Turner, $1962: 160$.

laquelle se forge pour le néophyte une nouvelle identité. Pourtant, ce que j'ai souhaité montrer ici, c'est que cette nouvelle identité se dessine dès les rites de séparation, au même titre qu'un certain nombre d'autres enjeux cruciaux du rituel grâce auxquels elle émerge: la mise en place de configurations relationnelles particulières, l'aptitude qu'ont les actes rituels à émouvoir, leur efficacité. En outre, lorsque différentes séquences de la séparation jouent sur le lien maternel comme sur l'ensemble des rapports entre novices, initiés, initiateurs et non-initiés, elles créent un contexte original qui permet de transformer par à-coups la relation mère-fils amenée à perdurer au-delà de l'initiation. Chez les Ndembu, Turner constatait que «there is no severance of the mother-son bond in Mukanda; only a change in character. Father and brother are the "figureheads" of a novice's "transition"[...] from infancy to manhood. But the family ties still hold throughout the change. The boys eventually go back to their mothers, but they return as men, though often merely little "men" of seven years old ". "De même chez les Seereer, le tout jeune initié regagne la maison de ses parents et y demeurera le plus souvent et selon le principe de la résidence patri-virilocale, une fois marié. Mère et fils continuent ainsi de se côtoyer, mais adoptent idéalement de nouveaux comportements l'un à l'égard de l'autre. Ce nouveau mode d'intersubjectivité qui leur échoie désormais se traduit par un système d'attitudes différent de celui de l'enfance et qui est la conséquence directe de l'évolution que le rite fait subir à l'attachement : y prédominent en public réserve et déférence. De mère d'un petit garçon, la mère du novice se retrouve potentiellement belle-mère et future grand-mère; son fils adulte quant à lui, est maintenant susceptible de devenir époux et père, et de nouvelles relations - d'affinité et de filiation - s'immiscent bien vite dans la dyade qu'ils formaient auparavant.

Dans les rites de séparation, on l'aura donc compris, tout est affaire de bonne distance, et certaines situations (la répartition des participants dans la maison pendant le muur par exemple) préfigurent d'ores et déjà l'éloignement des novices en brousse ainsi que les conditions futures de 
leur retour au sein de la concession familiale. La distance spatiale que l'on instaure lorsque les rites s'enclenchent se fera distance sociale à l'issue de l'initiation. Dans le même temps, et cela semble assez général dans le cas d'initiations masculines, ils jettent d'une manière ou d'une autre un regard en arrière vers la petite enfance, et en ce sens je voudrais souligner leur caractère rétrospectif. Les rites qui marquent le départ des novices insistent sur le lien maternel, on l'a vu, mais en utilisant et en manipulant certains motifs marquants du maternage (allaitement et nourrissage, portage, sommeil partagé), comme s'il fallait à tout prix pour en faire le deuil, réécrire et réinterpréter l'enfance dont on se défait. Les hommes et l'institution la remodèlent ainsi par fragments et de façon anarchique ou inversée comme pour brouiller la mémoire, rompre la familiarité et garantir qu'un fils une fois adulte ne sera pas tenté de " régresser " au contact de sa mère. À ce titre, il est intéressant de comparer l'initiation masculine aux rites féminins qui présentent pour leur part un aspect plutôt prospectif. Le ndut des femmes, dont les rites en pays seereer s'enchâssent dans ceux du mariage, est résolument tourné vers la vie conjugale. Aussi les étapes qui jalonnent le départ de la mariée vers la maison de son époux où elle sera initiée ne cherchent-ils pas tant à déconstruire le lien qui l'unit à sa mère qu'à poser d'emblée les bases de ceux qui l'unissent désormais à sa belle-mère. Notons qu'on n'observe pas non plus de situation qui serait inversement symétrique aux rites masculins : le couple père/fille ne fait pas l'objet d'un traitement particulier. La séparation est ainsi plus élaborée pour une mère et son fils qu'elle ne l'est pour une mère et sa fille - et ceci est avéré dans de nombreuses sociétés. Outre le fait qu'il faille marquer rituellement la distinction de sexe dans la relation de filiation, il s'agit de fonder les nouvelles positions de chacun dans la relation qui prévaudra à l'âge adulte des enfants, et cela avec d'autant plus de force que les intéressés poursuivent leur cohabitation audelà du rituel. À cet égard, remarquons que les pagnes, à la différence d'autres tissus que j'ai pu évoquer (pagnes teints à l'indigo pour les esclaves rituels; forok de sortie), suivent 
82 Weiner, 1992.

un circuit qui est finalement un peu à l'image du chemin qu'empruntent les fils lorsqu'ils se font initier. Ils reviennent à leur point de départ, la malle maternelle. Ces tissus, à l'instar des «inalienable possessions" dont parle Weiner ${ }^{82}$, sont en effet destinés à être conservés tandis que d'autres (pagnes noirs; tissus industriels) «partent». Comme le fils, ils restent chez la mère, ce qui n'est pas le cas du pagne de la mariée appelé moote. Fourni par la belle-mère, il change rapidement de mains au cours du mariage. Récupéré par la mère de la mariée, puis confié à une sœur ainée de celle-ci, le moote effectue ainsi un chemin inverse à celui de la fille.

Par ailleurs, si l'initiation seereer, aussi "édulcorée " soit-elle dans sa version actuelle, conserve aux yeux de tous une efficacité certaine, c'est qu'elle crée des situations particulièrement troublantes - paradoxes, jeux de regards et de dissimulation, jeux sur le lien maternel -, suscitant à mon sens deux registres d'émotions étroitement imbriqués. D'une part, les émotions tacites ou implicites sont celles qui sont révélées aux yeux de tous par des comportements spécifiques et attendus dans de tels contextes. On a vu que ces attitudes émotionnelles issues des relations qui se nouent pendant le rite se nourrissent les unes les autres soit à l'intérieur d'un ensemble de personnes relevant d'une même catégorie soit entre différentes catégories de participants. On pourrait aussi parler à cet égard d'émotions caricaturales ou stéréotypées que la répétition du rituel dans le temps ne fait que cultiver. D'autre part, les émotions d'ordre plus intime et que je qualifierais de mnésiques en ce sens qu'elles sont liées au vécu de chaque participant - expériences personnelles, souvenirs d'événements identiques passés - émergent de l'action rituelle dans son ensemble perceptible et des objets pris dans celle-ci. La vue des personnages voilés déclenche les souvenirs : un tel revivra sa propre initiation, une telle son mariage. Il va de soi que ces émotions sont de celles que l'ethnographe ne peut aborder que très superficiellement par le truchement du discours. Même très précisément mises en mots au cours d'entretiens, les émotions ainsi revisitées a posteriori, à la demande d'une étrangère qui plus est, perdent indubitable- 
ment de leur spontanéité et de leur authenticité. Le rapport entretenu par ces émotions, qu'elles soient "affichées " ou " ressenties », est un domaine qui reste largement à explorer et que je situerais intuitivement au cour des processus d'efficacité du rituel. Ici, c'est la convergence de l'assurance des hommes, du désarroi des mères, de la «disparition " des novices et de la peur des cadets tous ensembles associés au ressenti de chacun par rapport à l'action (indifférence, empathie, plaisir, crainte...) qui produit un effet tel qu'il rend seul et déjà efficace le processus initiatique et ce dès les rites préliminaires.

Au demeurant, j'ai montré comment les pagnes blancs étaient précisément au centre des enjeux émotionnels des rites de séparation. L'attention portée aux objets peut de ce fait être un excellent moyen d'aborder la question des affects dans le rituel. Le pagne, en tant qu'objet qui investit, créé une image efficace, véritable point de force du rituel, de telle manière que la convergence du fait d'en avoir été le témoin en tant que participant et d'en avoir été voilé pour les novices, pourrait à la limite être le minimum requis pour faire d'un jeune garçon un initié. Qui plus est, on a vu que les vertus protectrices que l'on prête à ces étoffes présentent en réalité plusieurs facettes - objet-médiateur et médiumnique, objet-bouclier et bâillon - relatives aux points de vue que les participants ont sur l'action et aux relations qu'ils entretiennent. C'est ce qui fait finalement du pagne un objet " piège » ou " miroir " qui capte et reflète à la fois toute une gamme d'états émotionnels. Son pouvoir d'évocation est tel que son emprise sur le corps de l'initiant ne laisse personne indifférent, même le moins impliqué des spectateurs. Si pour les initiateurs seereer ce sont avant tout les épreuves corporelles douloureuses de la période liminale qui font "rentrer le ndut " dans la tête des njuli, je pense également que les émotions liées au recouvrement rituel marquent à long terme le novice. Le pagne œuvre dans ce sens : la transformation que subit le garçon n'est donc pas simplement due aux épreuves initiatiques, elle est le fait des transformations relationnelles et des émotions que le pagne suscite au cours de la séparation. 
${ }^{83}$ Bonnemère, 2004 :

11.

$84 \mathrm{Ibid}: 14$

85 Ibid. : 74
Je conclurai enfin sur une remarque. Comme l'ont montré Pascale Bonnemère et les auteurs des contributions réunies dans l'ouvrage qu'elle a dirigé sur le rôle des femmes dans certains rituels masculins en Papouasie Nouvelle-Guinée, les initiations masculines ne concernent pas exclusivement des hommes et des garçons ${ }^{83}$. Le choix du pagne - objet féminin - comme moyen d'aborder l'initiation a orienté mon regard et a permis de prendre en compte la participation des femmes, les mères des novices en particulier, aussi sérieusement que celle des hommes. Et très rapidement, vus sous cet angle, on ne peut plus simplement dire que pour devenir des hommes adultes, les garçons doivent d'abord être séparés de leurs mères et que c'est là le seul processus que le rituel met en actes. On ne peut que constater avec Pascale Bonnemère, que bien qu'une telle affirmation soit sûrement vraie, elle est insuffisante, puisque ce qui semble réellement se jouer-là n'est pas seulement l'accession du garçon au statut de sujet en soi, mais une redéfinition de toute la configuration relationnelle autour de lui ${ }^{84}$. Et elle ajoute : "looking at what women do during male initiations certainly adds a crucial dimension to the analysis of these rituals. [...] it also has crucial implications for the future of the dayly relations between the boys and their mothers ${ }^{85}$." 


\section{Références bibliographiques}

Appadurai, A. (dir.)

1986 The Social life of things. Commodities in cultural perspective, Cambridge, Cambridge University Press.

Bazin, J.

2004 "Le roi sans visage ", L'Homme 170, pp. 11-24.

Bonnemère, P. (dir.)

2004 Women as unseen characters. Male ritual in Papua New Guinea, Philadelphia, University of Pennsylvania Press (Social Anthropology in Oceania).

Bonnot, $\mathrm{T}$.

2002 La Vie des objets. D'ustensiles banals à objets de collection, Paris, Éditions de la Maison des Sciences de l'Homme/ Mission du patrimoine ethnologique (Coll. Ethnologie de la France).

Bourdieu, P.

1982 "Les rites comme actes d'institution", Actes de la recherche en sciences sociales 43, pp. 58-63.

Breton, S.

2002 "Présentation. Monnaie et économie des personnes ", L'Homme 162, Questions de monnaie, [En ligne. URL: http://lhomme.revues.org/ document154.html. Consulté le 16 novembre 2006].

Coquet, $\mathrm{M}$.

2001 [1991] Textiles africains, Paris, Adam Biro (Textures).

Crétois, L. (R. P.)

1972-1977 Dictionnaire Sereer-Français, Dakar, CLAD (Les Langues nationales au Sénégal, 48).

Dione, S.

1983 L'Éducation traditionnelle à travers les chants et les poèmes seereer, Dakar, UCAD, thèse de doctorat de $3^{\mathrm{e}}$ cycle.

2004 L'Appel du ndut ou l'initiation des garçons seereer, Dakar, IFAN/ ENDAEditions (Patrimoine, 1-2).

Diouf, M.

1994 Sénégal, les ethnies et la nation, Paris, L'Harmattan/ UNRISD-Forum du Tiers-Monde.

Droogers, A.

1980 The Dangerous journey. Symbolic aspects of boy's initiation among the Wagenia of Kisangani, Zaire, The Hague / Paris / New-York, Mouton (Change and Continuity in Africa).

Dupire, M.

1978 "Rôle rituel du père et de la mère chez les Sereer Sin bilinéaires du Sénégal ", in Systèmes de signes, Paris, Hermann, pp. 111-122. 
Faye, Amade

1997 Le Thème de la mort dans la littérature seereer. Essai, Dakar, Les Nouvelles Éditions Africaines/ ACCT.

Foster, R.

1993 «Dangerous circulation and revelatory display: exchange practices in a New Ireland society " in J. Fagans (dir.), Exchanging Products, Producing Exchange Sydney, University of Sydney (Oceania Monograph, 43), pp. 15. 31.

Gamble, D. P.

1957 The Wolof of Senegambia. Together with notes on the Lebu and the Seereer, London, International African Institute (Ethnographic Survey of Africa, Western Africa, Part XIV).

Gravrand, H. (R.-P.)

1973 "Le symbolisme Sérèr ", Psychopathologie africaine, IX (2), pp. 237-265.

1990 La Civilisation Sereer, Pangool. Le génie religieux Sereer, Dakar, Nouvelles Éditions Africaines.

Houseman, Michael

1986a «Le mal pour le mâle : un bien initiatique ", in J. Hainard \& R. Kaehr (dir.), Le Mal et la douleur, Neuchâtel, Musée d'ethnographie, pp. 89-99.

1986b "Note sur quelques propriétés générales de la transformation initiatique ", Nouvelle revue d'ethnopsychiatrie, 6, pp. 31-40.

1993 "The interactive basis of ritual effectiveness in a male initiation rite", in P. Boyer (dir.), Cognitive aspects of religious symbolism, Cambridge, Cambridge University Press, pp. 207-225.

1999 "Quelques configurations relationnelles de la douleur ", in F. Héritier (dir.), De la violence, Paris, Odile Jacob, (II), pp. 77-112.

Juillerat, B.

1999 «Séparation, retour, permanence. Le lien maternel dans le rite naven des

Kandji, A. Y. Iatmul ", L'Homme 151, pp. 151-180.

1982 «Éducation hier et aujourd'hui chez les Wolofs ", Les Cahiers du mythe 7, pp. 15-25.

La Fontaine, J. S.

1985 Initiation. Ritual drama and secret knowledge across the world, Manchester, Manchester University Press (Themes in Social Anthropology).

Latry, M.-C.

1996 "Les couturières de la nuit", Terrain 26, in dossier Rêver [En ligne, mis en ligne le 21 juillet 2005. URL: http://terrain.revues.org/ document3149.html. Consulté le 7 décembre 2006].

Moisseeff, M.

1987 «Entre maternité et procréation : l'inceste », Patio Psychanalyse (nouvelle Série) 7, pp. 121-145.

1996 "Devenir père ou mère : un enjeu des rites de la puberté ", Générations. Revue française de Thérapie familiale 8, pp. 30-33. 
Ndiaye, A.

1982 «Un aspect de l'initiation chez les Wolofs : la circoncision », Demb ak Tey, Les Cahiers du mythe 7, pp. 26-30.

Ndiaye, R.

1986 La Place de la femme dans les rites au Sénégal, Dakar, Nouvelles éditions africaines (Traditions orales).

Paulme, D.

1976 La Mère dévorante. Essai sur la morphologie des contes africains, Paris, Gallimard (Tel).

Rabain, J.

1994 [1979] L'Enfant du lignage. Du sevrage à la classe d'âge, Paris, Payot Schneider, J. (Bibliothèque scientifique).

1987 "The anthropology of cloth ", Annual Review of Anthropologgy 16, pp. 409. 449.

Schneider, J. \& A. B. Weiner

1986 "Cloth and the organization of human experience ", Current Anthropology 27 (2), pp. 178-184.

Senghor, L. S.

1990 [1964] Euvre Poétique, Paris, Éditions du Seuil.

Stern, D. N.

1999 [1985] Le Monde interpersonnel du nourrisson. Une perspective psychanalytique et développementale, Paris, PUF (Le fil rouge).

Turner, V. W.

1962 "Three symbols of passage in Ndembu circumcision ritual. an interpretation ", in M. Gluckman (dir.), Essays on the ritual of social relations, Manchester, Manchester University Press, pp. 124-181.

1967 The Forest of symbols. Aspects of Ndembu ritual, Ithaca, New York, Cornell University Press.

1987 "Betwixt and between : the liminal period in rites of passage ", in L. Carus Mahdi, S. Foster \& M. Little (dir.), Betwixt and between : patterns of masculine and feminine initiation, La Salle, Ill., Open Court Publishing Company, pp. 5-19.

Van Gennep, A.

1981 [1909] Les Rites de passage, Paris, Éditions A. et J. Picard.

Weiner, A. B.

1989 "Why cloth? Wealth, gender, and power in Oceania", in A. B. Weiner \& J. Schneider (dir.), Cloth and human experience, Washington, Londres, Smithsonian Institution Press, Wenner-Gren Foundation for Anthropological Research, pp. 33-72.

1992 Inalienable possessions. The paradox of keeping-while-giving, Berkeley,Los Angeles,Oxford, University of California Press. 
Winnicott, D. W.

1975 [1971] Jeu et réalité. L'espace potentiel, Paris, Gallimard (Folio Essais).

Zempléni, A.

1991 «Initiation ", in P. Bonte \& M. Izard (dir.), Dictionnaire de l'ethnologie et de l'anthropologie, Paris, PUF, pp. 375-377. 